\title{
Gradhiva
}

\section{La description graphique. Dessins et photographies dans les carnets de terrain et le travail de conservateur de Henry Balfour}

The Graphicalization of Description: Drawing and Photography in the Fieldwork Journals and Museum Work of Henry Balfour

\section{Christopher Morton}

Traducteur : Camille Joseph

\section{(2) OpenEdition}

Journals

\section{Édition électronique}

URL : http://journals.openedition.org/gradhiva/3511

DOI : 10.4000/gradhiva.3511

ISSN : 1760-849X

Éditeur

Musée du quai Branly Jacques Chirac

Édition imprimée

Date de publication : 23 mai 2018

Pagination : 58-89

ISBN : 978-2-35744-110-1

ISSN : 0764-8928

\section{Référence électronique}

Christopher Morton, «La description graphique. Dessins et photographies dans les carnets de terrain et le travail de conservateur de Henry Balfour », Gradhiva [En ligne], 27 | 2018, mis en ligne le 23 mai 2020, consulté le 06 janvier 2021. URL : http://journals.openedition.org/gradhiva/3511 ; DOI : https:// doi.org/10.4000/gradhiva.3511 
biel + stone.

cup. 


\section{La description graphique}

Dessins et photographies dans les carnets de terrain et le travail de conservateur de Henry Balfour

par Christopher Morton

Cet article explore les échanges fluides entre le dessin et la photographie dans le travail du premier conservateur du Pitt Rivers Museum de l'université d'Oxford, Henry Balfour (18631939). En étudiant de près deux carnets de terrain compilés dans les années 1920 par Balfour lors de missions dans le nord-est de l'Inde et en Afrique de l'Est, j'examine les usages distincts et complémentaires du dessin et de la photographie, tant sur le terrain que dans le document plus formel du carnet. II s'agit plus généralement de mettre en lien le rapport de Balfour au document visuel avec sa formation aux sciences naturelles reçue dans les années 1880 , et de comprendre sa pratique au regard des approches anthropologiques du xIX siècle et de la manière dont, à cette époque, étaient envisagées les relations entre la collecte de données textuelles, dessinées et photographiques et leur présentation dans le musée. 
1. Ce texte est issu d'une communication présentée à la journée d'étude «Sur le vif. Méthodes et pratiques de terrain", dans le cadre du projet Photographie et dessin en anthropologie (1920-1950), organisée par Camille Joseph et Anaïs Mauuarin à l'Institut national d'histoire de l'art à Paris en 2016. Je tiens à remercier les organisatrices d'avoir pris l'initiative de cette journée à l'origine de la recherche présentée ici. Je voudrais également remercier les évaluateurs de mon texte pour leurs suggestions utiles.

2. On sait que ces journaux ont été régulièrement compulsés après la mort de Balfour. Celui-ci y faisait déjà référence dans les registres d'acquisition du musée, indiquant à ses collègues les passages où ils trouveraient davantage de renseignements sur tel ou tel objet.

À la date du 26 janvier 1931 du registre, par exemple, il écrit : "J'ai assisté à la fabrication de ce pot, voir notes dans mon journal H. Balfour. "

3. J'ai choisi de me concentrer sur ces deux carnets parce qu'ils datent de la période retenue pour le présent numéro, et qu'ils sont particulièrement représentatifs des pratiques de Balfour.
Cet article ${ }^{1}$ examine l'usage du dessin et de la photographie dans deux carnets de Henry Balfour (1863-1939), anthropologue et premier conservateur du Pitt Rivers Museum de l'université d'Oxford. Si j'envisage ces notes manuscrites comme des journaux de terrain, Balfour et nombre de ses contemporains les considérant d'ailleurs comme tels, il faut d'emblée souligner qu'ils n'ont en réalité pas grand-chose à voir avec les carnets à usage plus personnel des ethnographes de l'ère post-malinowskienne. En effet, ceux dont il est question ici étaient vraisemblablement conservés avec la documentation muséale et mis à disposition du personnel du musée pour faciliter ses recherches, et notamment vérifier certaines informations relatives aux objets collectés sur le terrain - leur contexte culturel, la manière dont ils étaient utilisés et les conditions de leur acquisition ${ }^{2}$. En effet, Balfour et les anthropologues de son époque ne faisaient pas de distinction très nette entre leurs recherches personnelles et les notes à usage privé d'un côté, et, de l'autre, le travail de collecte et de documentation pour le musée. Mais surtout, cette question n'avait pas nécessairement de sens pour Balfour: sa recherche se confondait avec celle du musée, les objets du musée étaient ceux qu'il avait collectés et documentés. Cette absence de séparation claire entre les missions effectuées à titre personnel ou institutionnel est plus caractéristique de l'anthropologie de musée du XIX ${ }^{\mathrm{e}}$ siècle que de la discipline universitaire, fondée sur un travail plus individuel, qui émerge précisément dans les années 1920. Pour les conservateurs formés dans les années 1880, comme Balfour, cette confusion restait dominante, faisant converger les identités individuelles et institutionnelles au sein d'un réseau de plus en plus vaste centré autour du musée, de ses donateurs, des étudiants et des chercheurs: un musée relationnel dont la mission consistait à collecter tant les individus que les objets (Gosden et Larson 2007 : 5).

Cet article propose différents éclairages sur la manière dont Balfour utilisa les données visuelles compilées dans deux journaux datant des années $1920^{3}$ - le premier rapporté de son voyage dans les Naga Hills en 1922 et le second de son séjour au Kenya et en Ouganda en 1928. Nous nous intéresserons d'abord à l'influence de sa formation en sciences naturelles sur ses observations ethnographiques, ses descriptions et les expositions qu'il organisa au Pitt Rivers Museum. Dans un second temps, nous montrerons que Balfour, comme de nombreux anthropologues, continua au cours du $x x^{e}$ siècle à dessiner directement ce qu'il observait sur le terrain, et nous nous interrogerons sur la valence et la pérennité de ces dessins en tant que preuves scientifiques. II s'agira ensuite de comparer les usages du dessin et de la photographie, de comprendre dans quels cas on jugeait qu'il était plus approprié d'utiliser l'un ou l'autre médium et de saisir la valeur scientifique supposée de chacun. Enfin, nous nous demanderons dans quelle mesure les questions d'objectivité et de subjectivité soulevées par les méthodes de terrain de l'époque sont (ou étaient) particulièrement décisives, l'urgence étant à l'époque à la traduction culturelle [cultural translation] dont était chargé le «tisseur » expérimenté du savoir anthropologique, dans le cadre didactique du musée ethnographique.

\section{L'anthropologue comme tisseur}

Henry Balfour est né à Croydon en 1863. À Oxford, il étudia les sciences naturelles et se spécialisa dans la biologie et la morphologie animale, avant d'obtenir son diplôme en 1885. Parmi ses professeurs figuraient 
Henry Nottidge Moseley, John Obadiah Westwood (entomologiste et paléographe) et Edward Bagnall Poulton (un évolutionniste enseignant la zoologie). Titulaire de la chaire Linacre d'anatomie humaine et comparée depuis 1881 , Moseley allait exercer une influence considérable sur la pensée de Balfour. II se rendit à Ceylan en 1871 et rejoignit plus tard l'équipe du HMS Challenger de la Royal Navy pour une expédition de quatre ans autour du monde, de 1872 à 1876, au cours de laquelle Moseley accumula une masse considérable d'informations sur les peuples indigènes rencontrés pendant le voyage. C'est aussi en partie grâce à lui que, en 1884, l'université d'Oxford accepta d'acquérir l'importante collection ethnographique d'Augustus Pitt Rivers, dont Moseley devint responsable un an plus tard. À la recherche de jeunes étudiants soigneux et appliqués pour l'aider dans l'immense tâche de mettre en place les nouvelles vitrines d'exposition, Moseley se tourna vers Walter Baldwin Spencer et Balfour. Mais il prévint ce dernier:

II s'agira de menus travaux en tous genres, faire des petits dessins, rédiger et taper soigneusement à la machine des cartels, écrire des descriptions pour le catalogue, arranger les vitrines, réparer, regrouper, nettoyer, aider le menuisier à fixer des choses sur les panneaux, rechercher des objets de toutes sortes dans des livres illustrés, les voyages de Cook, etc 4 .

À l'origine, Balfour n'avait qu'un contrat d'un an, mais il entreprit très vite de négocier un emploi permanent auprès de l'université. En 1889, il devint "sous-conservateur du Pitt Rivers Museum » et fut nommé conservateur à la fin de l'année 1890, bénéficiant du même statut que les professeurs travaillant au musée. II conserva ce poste jusqu'à sa mort, à l'âge de soixante-quinze ans, en 1939.

À la fin de sa carrière, Balfour se définissait comme un «technologiste comparatiste" [comparative technologist]. La comparaison était d'abord spatiale, car il s'intéressait aux questions d'invention, de mutation, de diffusion et de dissémination des techniques et de la forme des objets. Mais elle était également temporelle: Balfour étudiait les différents stades culturels que traversent les sociétés et la possibilité pour les ethnologues de mettre au jour leur préhistoire. Selon lui, l'ethnographie australienne pouvait ainsi expliquer les cultures paléolithiques européennes. Cette approche comparative était intimement liée à son expérience concrète au sein du musée: il était chargé de l'organisation, de l'agencement et de la présentation des objets figurant dans les séries typologiques de Pitt Rivers, qu'il adapta très largement à ses propres conceptions. II alla jusqu'à y voir la preuve que les idées nouvelles se greffent à des idées déjà existantes et que les types d'objets sont, à l'échelle mondiale, liés entre eux comme les branches d'un arbre. II existait pour lui un lien très étroit entre la compréhension intellectuelle d'un artefact et la relation que celui-ci entretenait avec d'autres objets exposés, à tel point que Balfour dispensait généralement ses cours dans les galeries mêmes du musée ${ }^{5}$. Un ancien étudiant raconte ainsi :

Nos séances de travail avec Henry Balfour avaient toujours lieu au Pitt Rivers Museum, où il était conservateur, devant les vitrines auxquelles venaient souvent s'ajouter des spécimens présentés sur des plateaux ou en vrac... Les paquets de notes qu'il apportait pour ces cours itinérants faisaient penser à Darwin, qui utilisait le moindre bout
4. Pitt Rivers Museum Manuscript Collections, Pitt Rivers Museum Papers, boîte 1 (volume consacré à la création du musée), 11 octobre 1885

5. Il commença officiellement à enseigner à l'université d'Oxford en 1893, puis il continua ses cours et son travail de tutorat jusqu'à la création en 1905 d'un comité [Committee for Anthropology] et d'un diplôme d'anthropologie. II poursuivit parallèlement son enseignement dans le cadre du musée jusqu'à la fin des années 1930. 


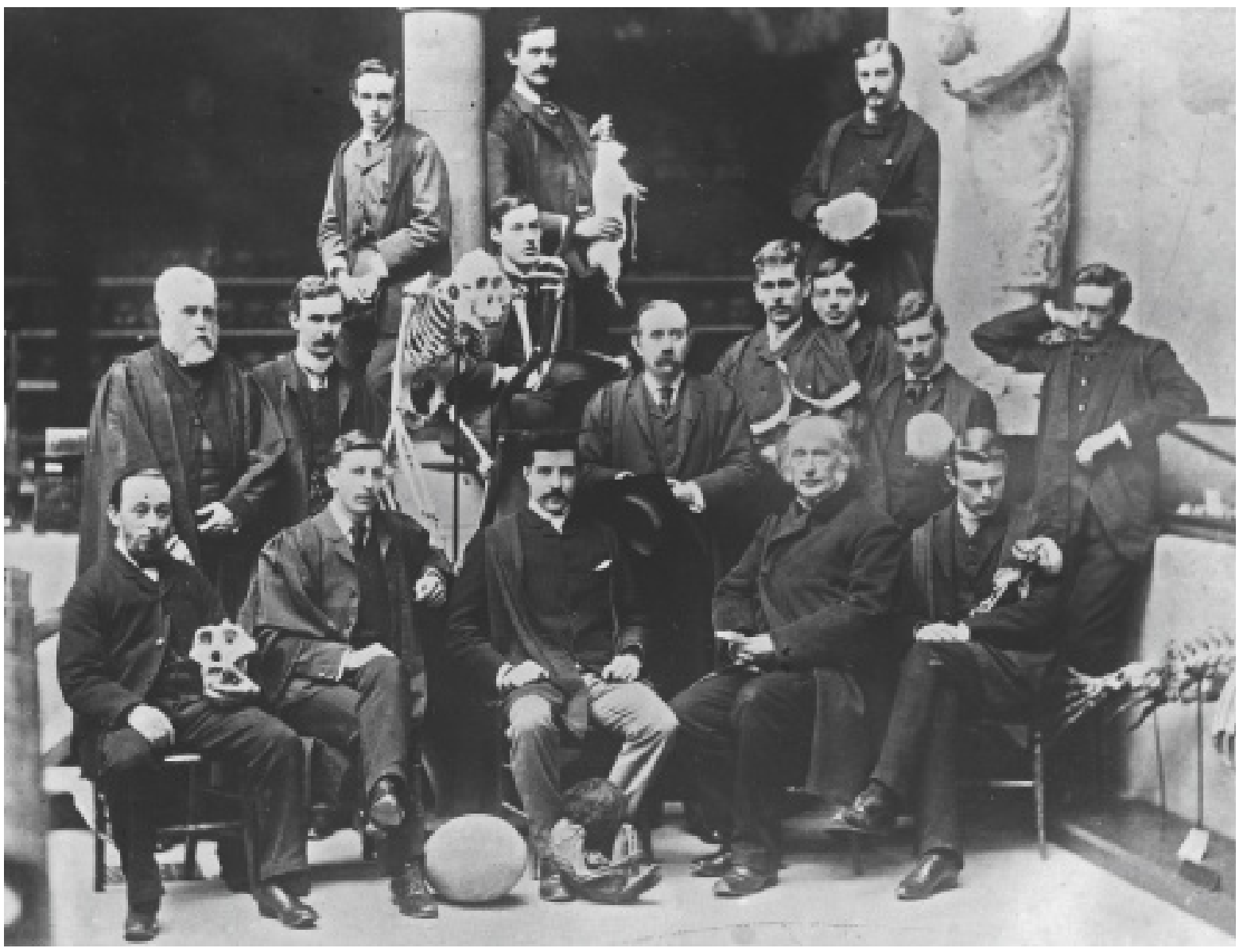

fig. 1

Anonyme, Membres de la classe d'anatomie humaine et de physiologie, Université d'Oxford, département de morphologie. Oxford

University Museum of

Natural History, juste avant es examens finaux.

Henry Balfour (tenant un lapin), Henry Nottidge Moseley, Baldwin Spence et Henry Acland, vers 1884, épreuve gélatino-

argentique. Avec l'aimable autorisation du Pitt Rivers

Museum, University of

Oxford, 1998.267.85. 
de papier: c'étaient des bouts de feuille de toutes les tailles et de toutes les formes où s'intercalaient parfois des articles de presse et des passages de lettres. (Wallis 1957: 786-787)

Si Balfour ne développa jamais de théorie sociologique à proprement parler, ses premiers travaux (voir par exemple Balfour 1890) témoignent de l'influence sur sa pensée de l'approche socioculturelle évolutionniste défendue par Augustus Pitt Rivers, des idées de «survivances» [survivals] culturelles développées par Edward Burnett Tylor ${ }^{6}$, ainsi que de l'anthropologie allemande. Évoquant à la fin de sa carrière ce qu'il considérait comme la relation symbiotique de l'enquêteur de terrain et de l'anthropologue de cabinet, il utilisait une analogie textile pour décrire les «fileurs d des données ethnographiques d'un côté et les «tisseurs » du savoir anthropologique de l'autre:

La responsabilité du fileur est donc de fournir des fils épais et bien identifiés (sous la forme de faits parfaitement attestés) au tisseur, qui les unifiera par un procédé textile et créera non seulement un tissu, mais aussi un motif en croisant la «chaîne» des faits avec la «trame » de l'argumentation. (Balfour 1938: 8)

En 1995, l'anthropologue Mary Douglas revenait sur le livre de Balfour, Spinners and Weavers [Fileurs et tisseurs]. Alors qu'elle cherchait à s'inscrire en doctorat d'anthropologie à Oxford, le successeur de Balfour au Pitt Rivers Museum, Thomas Kenneth Penniman, lui avait donné à lire l'ouvrage pour le lendemain. En constatant la division qu'établissait Balfour entre la collecte de données sur le terrain et le travail d'élaboration théorique, elle s'était alors demandé «si les faits peuvent exister sans la théorie» (Douglas 1995: 262). Cette question nous est aujourd'hui familière, mais pour Balfour et ses contemporains, la théorie ne pouvait être formulée qu'à partir du moment où étaient établies des comparaisons et des mises en relation interculturelles; elle ne devait pas influencer la collecte de données. Andrew Lang, spécialiste des études folkloriques, résume bien cette posture: «Les voyageurs et les missionnaires se sont mis à lire des livres d'anthropologie; par conséquent, les preuves qu'ils rassemblent risquent, aujourd'hui bien plus qu'auparavant, d'être biaisées par des théories anthropologiques. " (Lang 1901: 335)

Balfour « fila » notamment les faits anthropologiques à propos de l'évolution des arts décoratifs, une question qu'il aborda dans divers articles et conférences prononcées au début des années 1890 et qui donna lieu à sa seule monographie publiée (Balfour $1893^{7}$ ). Dans cet ouvrage, Balfour montre que la copie, plus ou moins fidèle, est le principal moteur de variation dans les motifs ${ }^{8}$. Elle mène à la dégénérescence mais aussi à l'innovation. De même, les motifs peuvent perdre leur signification au fil du temps, laissant des «survivances » de motifs relevant d'une étape culturelle bien antérieure. Je reviendrai sur cet intérêt pour la copie, présent dans les travaux de Balfour sur l'évolution de l'ornement, en abordant sa pratique de la photographie et du dessin de terrain. Mais cette attention au processus de copie renvoie également à des questions qui dépassent le cadre de cet article, à savoir les pratiques et les techniques institutionnelles de reproduction des images destinées à servir d'illustrations ou à être affichées dans les expositions du musée, dans lesquelles Balfour fut constamment impliqué.
6. Tylor décrit le concept de "survivances" comme "des processus, des coutumes, des opinions, etc., qui ont été transportés par la force de l'habitude dans un nouvel état social, différent de celui dont ils sont originaires, et [qui] subsistent dès lors comme des preuves et des exemples qu'il a existé une condition culturelle antérieure à partir de laquelle une nouvelle a évolué. » (Tylor 1871: 16)

7. Je remercie Eléonore Challine d'avoir attiré mon attention sur le fait que le livre de Balfour de 1893 parut la même année que l'ouvrage de l'historien de l'art allemand Aloïs Riegl consacré à l'histoire de l'ornementation, Stilfragen. Grundlegungen zu einer Geschichte der Ornamentik (Berlin, G. Siemens), sans que celui-ci ne soit toutefois mentionné par Balfour. Alfred C. Haddon publia lui aussi un ouvrage sur la question en 1895 (Evolution in Art: As Illustrated by the Life Histories of Designs, Londres, W. Scott Itd.).

8. Balfour 1890, 1893; Pitt Rivers Museum Manuscript Collections, Balfour Papers, boîte 4A. 
9. Quatre des dessins de Spencer [1903.39.82-85] furent exposés dans la vitrine d'Art aborigène du Pitt Rivers Museum entre 2009 et 2016 , avant d'être remplacés par des œuvres de l'artiste aborigène Christian

Thompson.
Lors de ses expéditions, Balfour emportait presque toujours avec lui des carnets et des journaux. Entre la fin des années 1880 et le début des années 1930, il consacra une grande partie de ses congés à voyager en Afrique, en Australie, en Inde, en Amérique du Sud ou encore en Scandinavie. Les deux journaux examinés ici sont particulièrement révélateurs d'un changement dans ses méthodes de travail sur le terrain. C'est aussi le moment où une nouvelle génération de spécialistes d'anthropologie sociale est formée à la London School of Economics, sous la houlette de Bronislaw Malinowski. J'ai consacré un grand nombre de travaux aux photographies de l'un des plus célèbres d'entre eux, Edward Evans-Pritchard, qui effectua plusieurs missions au Soudan du Sud dans les années 1920 et 1930. J'ai étudié les rapports complexes que la photographie entretient à cette époque avec les autres méthodes de terrain et les textes publiés, ainsi que la manière dont les populations locales jouent un rôle actif en infléchissant le type de prises de vue (Morton 2009). II existe par ailleurs de plus en plus de recherches consacrées au dessin de terrain à cette période. Dans son étude sur les dessins réalisés par Bernard Deacon pendant sa mission au Vanuatu en 1926-1927, Haidy Geismar a ainsi mis le doigt sur un certain nombre de ruptures entre textes et dessins dans le travail de Deacon, ce qui le conduisait parfois à une véritable «impasse visuelle». Dessiner représentait en effet pour Deacon "une forme de pensée (visuelle) et une pratique incarnée et située" (Geismar 2014: 105). II existe par ailleurs plusieurs points communs entre les croquis de Deacon figurant des dessins de sable au Vanuatu (ou des photographies de dessins réalisés par des informateurs) et la pratique des anthropologues de la génération de Balfour.

En 1903, Spencer, ancien camarade de classe de Balfour à Oxford, légua au musée une série de peintures grand format représentant des fresques sur sable qu'il avait pu voir lors de la cérémonie totémique wollunga des Warumungu dans la région australienne de Tennant Creek, située dans le Territoire du Nord. Tracés à même le sol et destinés à un rite spécifique, les motifs indigènes étaient par nature éphémères. En les copiant sur papier, Spencer créa une forme artistique hybride, ni tout à fait indigène, ni tout à fait européenne. En raison de leur nature très ambivalente, ces peintures purent être exposées comme objets d' "art aborigène" pendant de nombreuses années au Pitt Rivers Museum, à la fois en tant que motifs indigènes et documents historiques ${ }^{9}$. La fascination de Deacon pour les dessins de sable du Vanuatu et ses très nombreux croquis montrent qu'il avait saisi leur rôle dans la transmission de connaissances culturelles, tout comme le fait qu'ils sont une expression de la manière dont les indigènes «théorisent la connectivité et l'enchevêtrement des cultures" (ibid 2014: 109). Les dessins de terrain de Balfour partagent un certain nombre de caractéristiques avec les pratiques de ces anthropologues, immergés dans leur terrain sur de longues durées; mais ils sont aussi très différents dans la mesure où Balfour, qui ne restait jamais bien longtemps au même endroit, avait une approche plus superficielle, malgré une qualité d'observation et un œil attentif aux détails. En ce sens, il incarne les préoccupations et les pratiques visuelles d'un certain courant de l'anthropologie britannique, ancré dans les musées et intéressé par la question de la culture matérielle. 


\section{Nagaland, 1922}

Commençons par examiner le journal de terrain de Balfour datant de son voyage au Nagaland, en Inde du Nord, en 1922. Il y avait été invité par d'anciens étudiants d'Oxford, James Philip Mills (1890-1960) et John Henry Hutton (1885-1968), alors membres de l'administration coloniale. En janvier 1922, Mills écrivait à Balfour:

Quelle bonne nouvelle d'apprendre que vous allez vraisemblablement venir jusqu'aux Naga Hills à l'automne prochain. N'oubliez pas que je veux passer beaucoup de temps avec vous. Nous irons ensemble étudier les Nagas + les oiseaux. Je ne connais pas de livre sur l'ornithologie de la région, mais je pense que quand je serai rentré + aurai un peu de temps, je pourrai établir une liste relativement exhaustive des oiseaux sur lesquels j'ai pris des notes $[\ldots]^{\mathbf{1 0}}$.

II n'est pas surprenant que les oiseaux aient été au programme de Balfour: il s'y intéressa toute sa vie, et il n'est pas rare de trouver dans ses carnets des notes ou même des dessins de volatiles. Mills et Hutton organisèrent ensemble un circuit permettant à Balfour de «voir un peu chaque tribu ${ }^{\mathbf{1 1}}$ ». Pendant son séjour dans le Nagaland entre septembre et décembre 1922, les trois hommes travaillèrent sur leurs collections (cela faisait déjà longtemps que Mills et Hutton collectaient des objets pour le compte du musée). Mills voulait montrer sa collection à Balfour en personne avant de l'expédier à Oxford. Balfour passa la première partie de son séjour à voyager en compagnie de Hutton. Puis les deux hommes rejoignirent Mills, avec lequel Balfour poursuivit son circuit. Sur leur route, ils collectèrent des objets pour le musée. Mills fut chargé de préparer et d'envoyer en Angleterre la collection de Balfour après le départ de ce dernier ${ }^{\mathbf{1 2}}$.

Lors de ce voyage, Balfour avait semble-t-il toujours avec lui un petit carnet dans lequel il dessinait, écrivait et prenait des notes sur ses lectures. Le soir, il tenait son journal et faisait le récit de sa journée (les dessins étaient sans doute réalisés plus tard). Sur deux pages d'un carnet de terrain (fig. 2), on peut lire à gauche des notes prises sur un livre de T. H. Lewin décrivant des voyages en Inde, en particulier concernant la musique; sur la page de droite est représenté un joueur de pena de Manipour, que Balfour vit jouer le vendredi 29 septembre sur les pelouses de la demeure du Résident général à Imphal. Au dessin s'ajoutent des précisions sur la couleur des habits du musicien et même des différents pompons attachés à l'instrument. On trouve en bas de la page des essais de crayons de couleur, probablement faits au moment où Balfour préparait une version plus soignée du croquis dans un carnet cartonné grand format (fig. 3), dans lequel figurent également la pena et l'archet que Balfour précise avoir dessinés à l'échelle 1/7.

En comparant le croquis réalisé sur le vif par Balfour et la belle copie figurant dans son journal, on voit apparaître la forme finale de sa méthode d'observation et d'annotation. Mais le croquis du joueur de pena révèle aussi que Balfour accorde plus d'importance aux détails de la culture matérielle (y compris les habits) qu'à l'ensemble de la scène qui se déroule devant lui. La pose du musicien est de toute évidence une recréation artistique des mouvements que Balfour eut l'occasion d'observer à Imphal. Dès lors, ce dessin vise avant tout à décrire la manière dont on joue de la pena et insiste moins sur la figure du musicien lui-même. Dans la plupart des croquis,
10. Pitt Rivers Museum Manuscript Collections, Mills Papers, lettre à Balfour, 13 janvier 1922.

11. Pitt Rivers Museum Manuscript Collections, Mills Papers, lettre à Balfour, 6 juin 1922.

12. Pitt Rivers Museum Manuscript Collections, Mills Papers, lettres à Balfour, 23 novembre et 7 décembre 1922. 


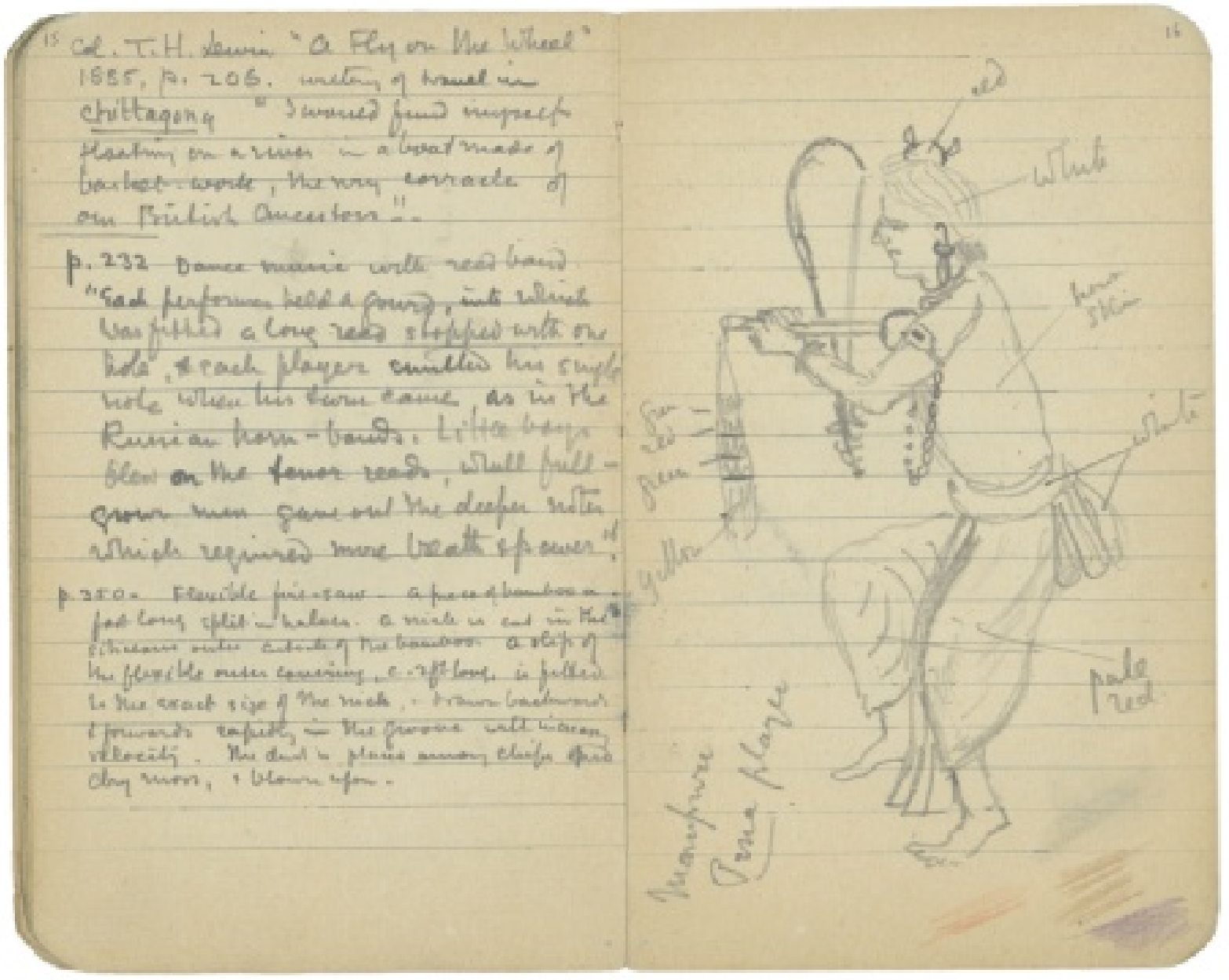

fig. 2

Double page (p.15-16)

d'un carnet de terrain

tenu par Henry Balfour

lors de son voyage chez

les Nagas. On y voit un

joueur de pena. Pitt Rivers

Museum Manuscript

Collections, Balfour

Papers, boîte 3, objet 3 .

Avec l'aimable autorisation

du Pitt Rivers Museum,

University of Oxford. 




fig. 3

Page 79 du volume 1 du journal de terrain de Henry Balfour chez les Nagas, montrant un joueur de pena et plusieurs dessins de cet instrument. Pitt Rivers Museum Manuscript Collections, Balfour Papers, boîte 3. Avec l'aimable autorisation du Pitt Rivers Museum, University of Oxford, Balf_1922NHills-Ass1_79-R. 


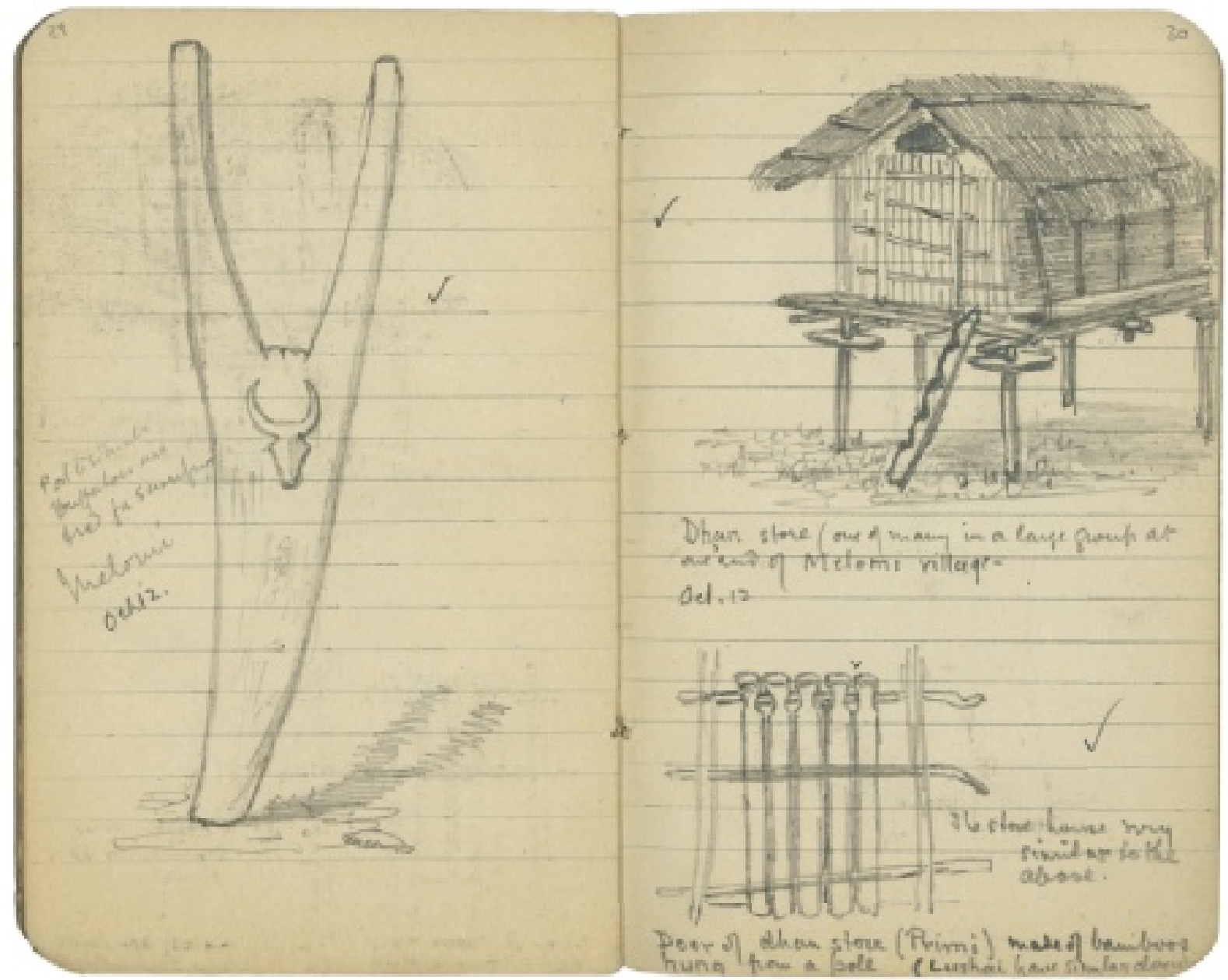

fig. 4

Double page (p. 29-30)

d'un carnet de terrain de

Henry Balfour dans le

Nagaland. Dessin d'un

poteau utilisé notamment

pour des sacrifices de

buffles. Pitt Rivers Museum

Manuscript Collections,

Balfour Papers, boîte 3.

Avec l'aimable autorisation

du Pitt Rivers Museum,

University of Oxford. 


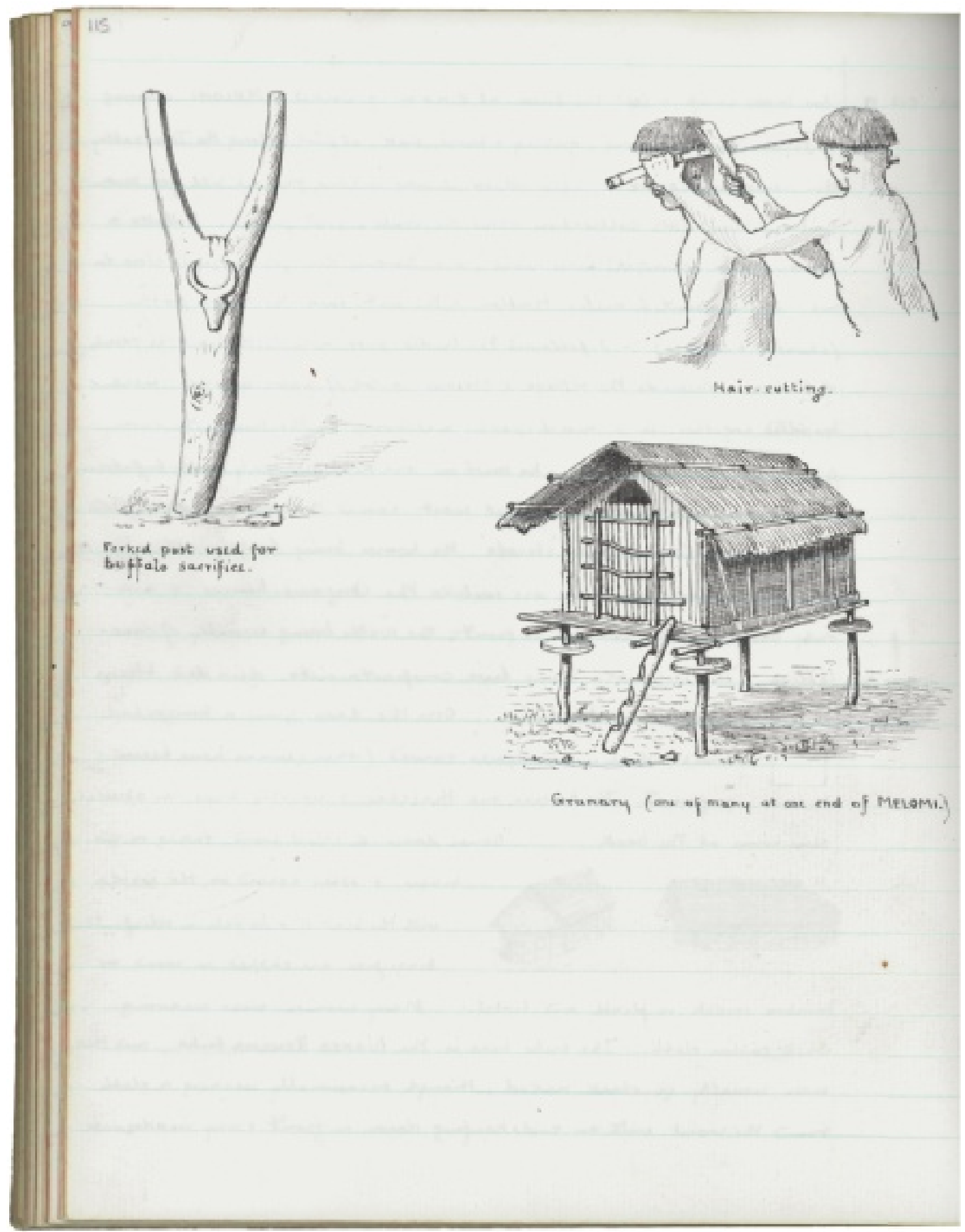

fig. 5

Page 115 du volume 1 du journal de Henry Balfour dans le Nagaland. Dessin montrant un poteau utilisé notamment pour les sacrifices de buffles. Pitt Rivers Museum Manuscript Collections, Balfour Papers, boîte 3 Avec l'aimable autorisation du Pitt Rivers Museum, University of Oxford, Balf_1922NHills-Ass1_115-R. 
13. II s'agit de films 124 de chez Kodak de format $8 \times 11 \mathrm{~cm}$, sur lesquels était automatiquement inscrit en haut un numéro. Les photographies furent très vraisemblablement prises avec un Brownie $n^{\circ} 3$ à soufflet. II est probable que Balfour ait acheté cet appareil en 1905 (peu de temps après sa commercialisation), avan son voyage en Afrique du Sud, car les négatifs issus de cette dernière expédition ont le même format. Le viseur Watson fut peut-être endommagé durant le voyage au Nagaland, entraînant des problèmes de cadrage. Les négatifs de 1928 mesurent $6 \times 8 \mathrm{~cm}$ et correspondent là encore sans doute à un Kodak, peut-être un autre Brownie ou un appareil de poche à soufflet.

\section{ci-contre}

fig. 6

Page de dessins,

"Différentes têtes de sanglier sculptées sur les façades des maisons Angami ». Pitt Rivers Museum Manuscript Collections, Balfour

Papers, boîte 3, fichier 2. Avec l'aimable autorisation du Pitt Rivers Museum,

University of Oxford. les individus servent uniquement à remettre en contexte une parure, un ornement ou des objets, Balfour s'intéressant peu à la description concrète des types raciaux ou de la vie sociale. Dans une autre page du carnet, on trouve des notes sur l'organisation de l'espace dans une maison naga et sur la méthode de construction, complétées par le dessin d'une sculpture représentant la tête d'un gayal (ou mithan, c'est-à-dire Bos gaurus frontalis) ornant l'entrée d'une habitation. Sur la page d'en face, des croquis faits sur le vif illustrent la manière de porter une ceinture d'écorce serrée et une parure de tête. Une fois ces croquis reportés dans la version plus formelle de son journal, Balfour faisait une petite croix près du dessin de départ pour se rappeler qu'il l'avait copié. Dans l'exemple figurant sur la figure 4, le dessin d'un poteau sculpté en forme de fourche, utilisé lors des sacrifices de buffles, et un grenier surélevé servant à conserver les récoltes ont été recopiés dans le journal avec un troisième dessin, qui témoigne de la manière dont les Naga coupent les cheveux à l'aide de deux lames dao (fig.5).

Ces versions travaillées des croquis de terrain n'étaient pas seulement destinées au journal de Balfour. Certaines étaient recopiées sur un papier de luxe et fixées sur un carton ou bien encadrées pour être exposées dans le musée avec les nombreux artefacts collectés par Hutton et Mills. Sur un dessin ayant vraisemblablement été exposé dans le musée, Balfour avait rassemblé plusieurs croquis réalisés au cours de son voyage et montrant différents motifs sculptés de têtes de sanglier fixés sur les façades des maisons de la tribu des Angami (fig. 6). Comme je l'ai rappelé plus haut, Balfour s'intéressait tout particulièrement à la manière dont les motifs artistiques deviennent de plus en plus stylisés par rapport à leur référent naturel. Dans ce cas précis, il les a ordonnés de manière à suggérer la dégénérescence du motif depuis une version naturaliste à gauche, jusqu'à une autre stylisée, à droite. En ligne avec l'évolutionnisme socioculturel qui caractérise sa formation et son approche, le savant envisageait d'abord les rapports entre ces objets, provenant d'époques et d'espaces différents, sous l'angle d'une comparaison formelle.

Balfour ne se contenta pas de dessiner la culture matérielle des Naga, il utilisa aussi un appareil photographique pour garder la trace, par exemple, d'un ornement de façade particulièrement complexe. Mais ses photographies prises au Nagaland sont de mauvaise qualité, soit parce que le viseur était défectueux, soit parce qu'il ne savait pas très bien se servir de son appareil. Bon nombre des négatifs sont mal cadrés, le sujet central apparaissant trop haut dans le cadre ou bien dans un angle ${ }^{13}$. Sur la plupart des négatifs défectueux, Balfour traça directement un nouveau cadre pour tenter de «sauver» des détails utiles ou éliminer au contraire des éléments superflus. Ainsi, par exemple, il recadra un cliché pour effacer l'ombre du casque qu'il portait alors, rendant du même coup invisible tout le contexte colonial de la production de cette image. Sur d'autres photographies, les traits tracés sur l'image servent à modifier l'orientation et le cadrage. On remarquera comment les lignes (tracées à l'encre au revers du négatif) éliminent du cadre de la figure 7 la femme située à gauche de l'image: cela s'explique en partie parce qu'on ne peut pas voir ce qu'elle a dans le dos, mais aussi parce qu'elle ne participe pas directement du sujet central de l'image. Ce nouveau cadrage, indiquant au photographe du musée la façon dont l'épreuve devait être tirée, établissait de nouvelles relations spatiales entre les sujets photographiés et avait du même coup pour effet de créer une nouvelle image répondant à des choix différents en termes d'esthétique et de composition. 


$$
\begin{array}{lll}
5 & 15 \\
4 & 18
\end{array}
$$




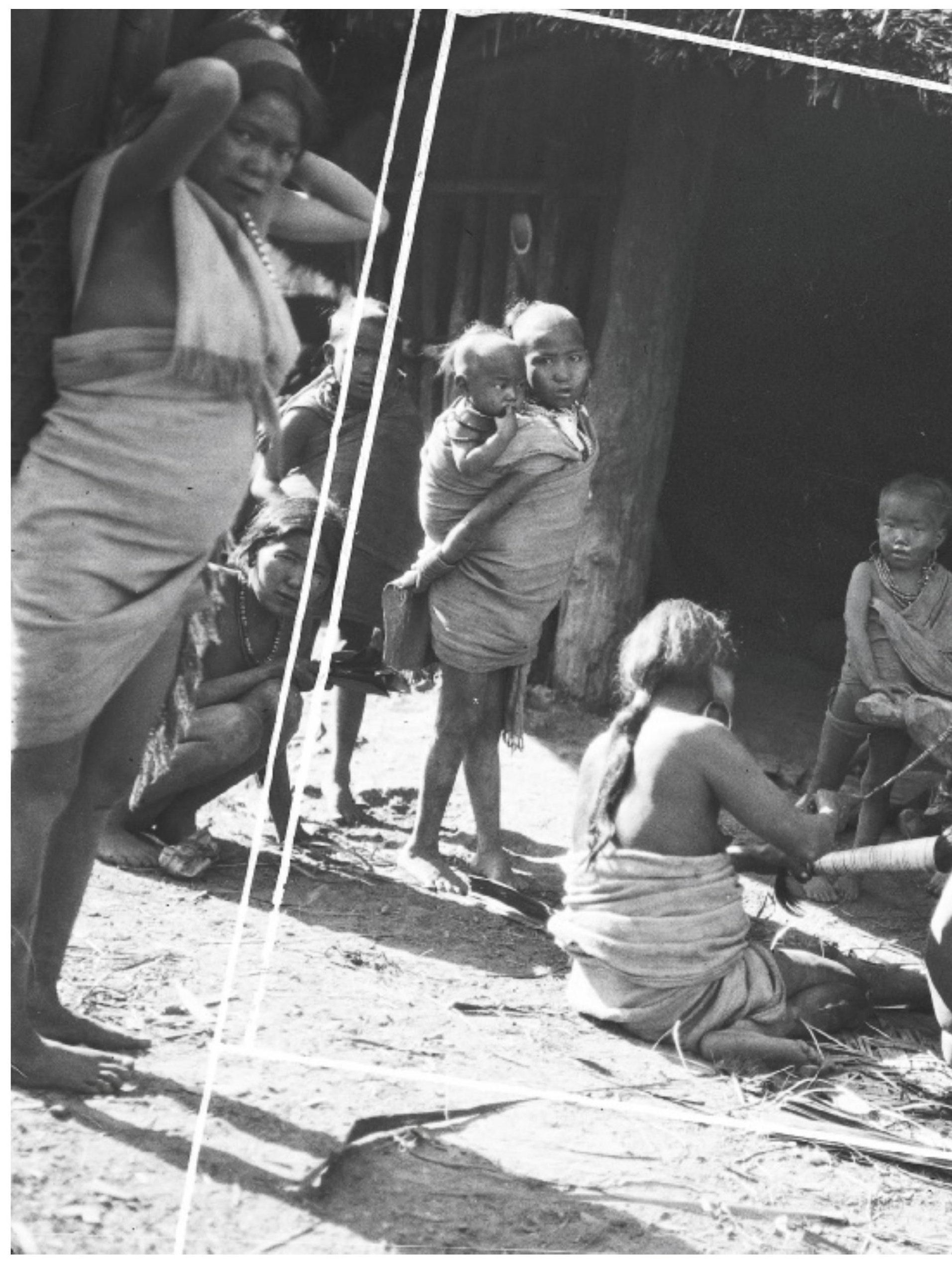




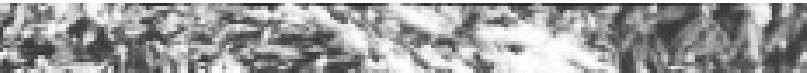
3.7



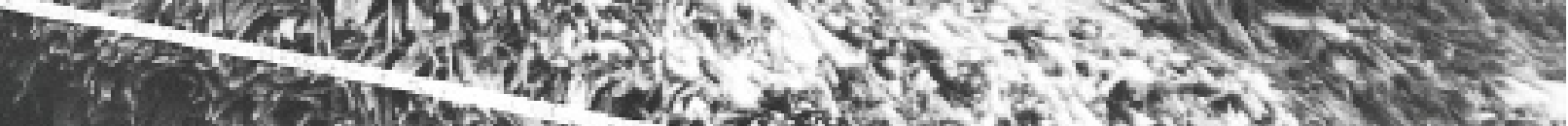

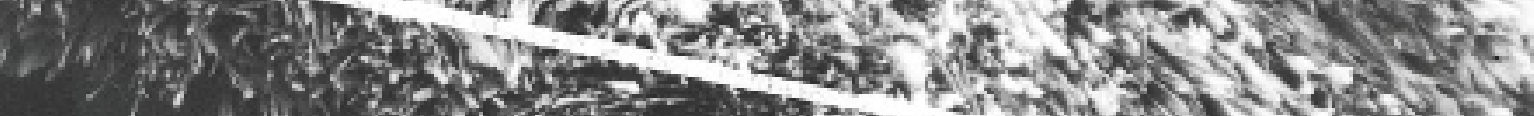

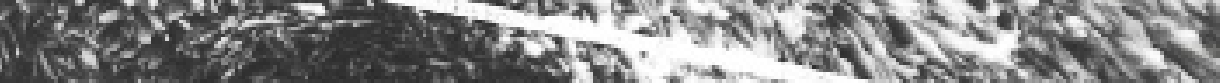

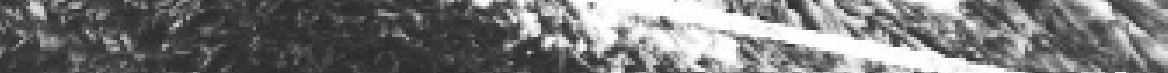

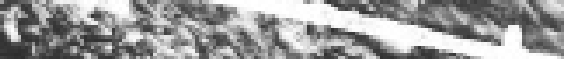

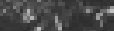

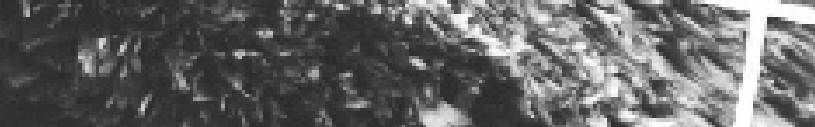

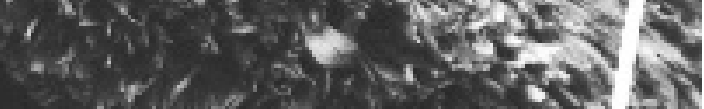

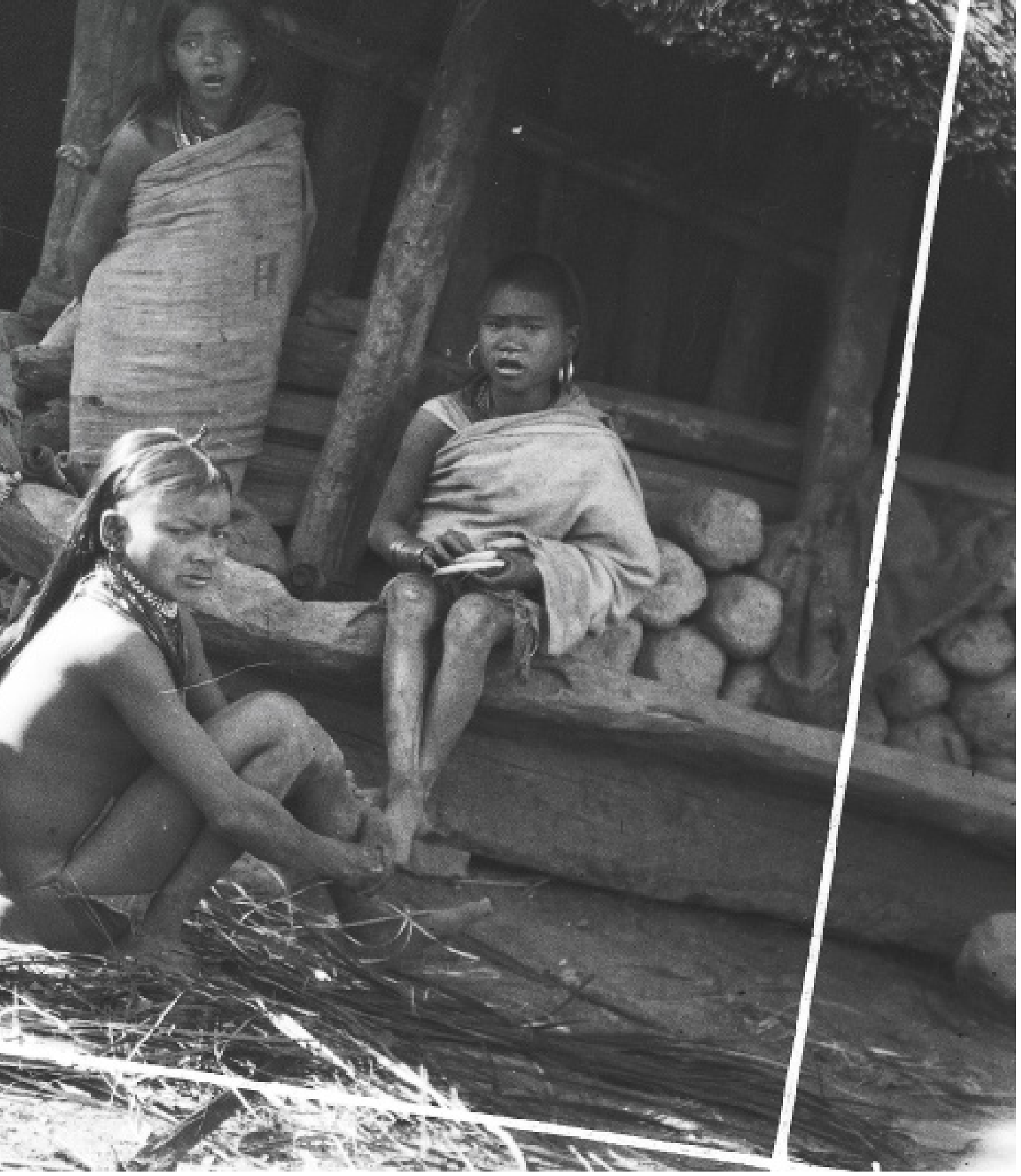




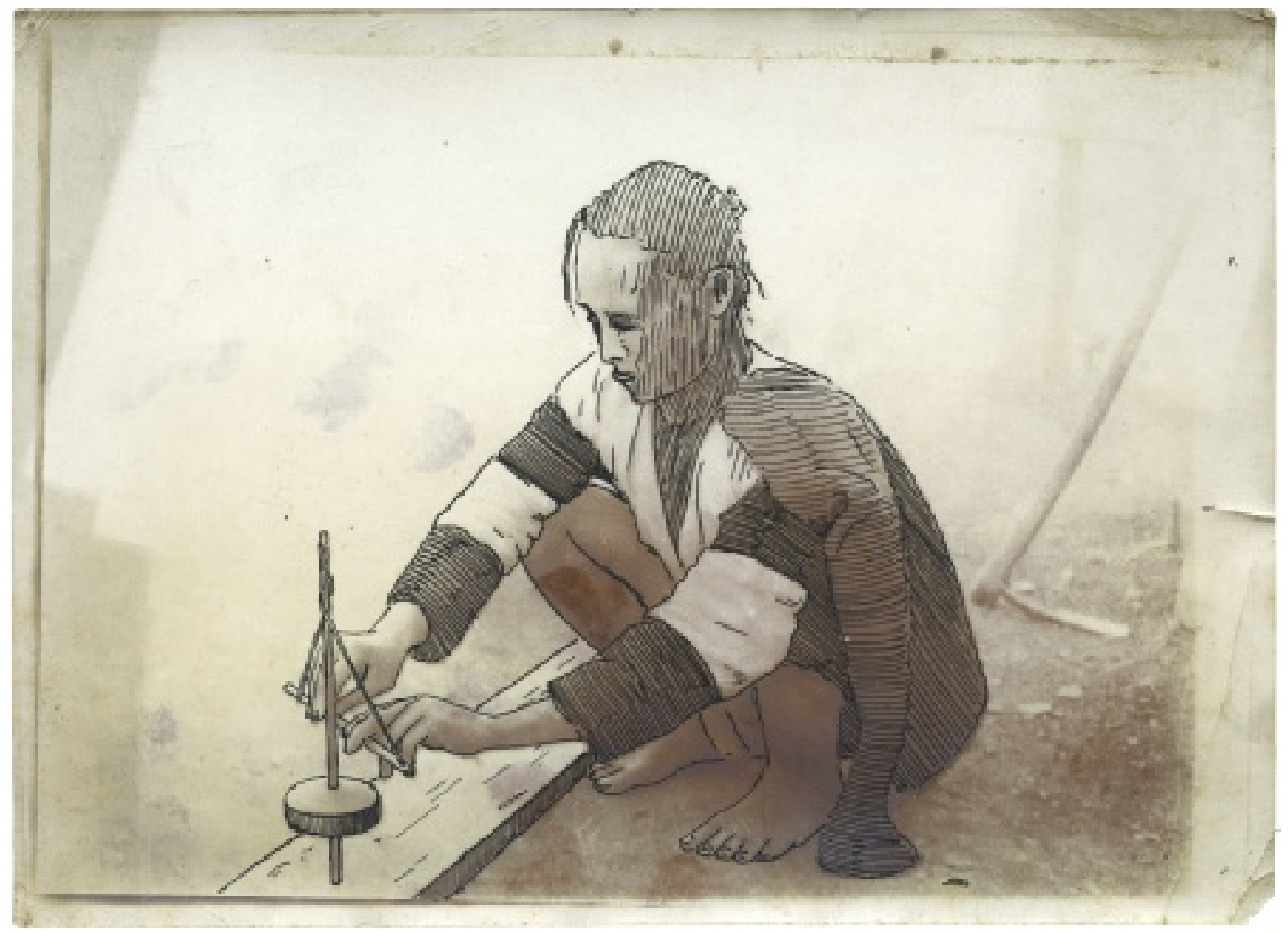

fig. 8

Photographie mal exposée montrant une femme Naga ou Manipuri faisant du feu. Henry Balfour a dessiné par-dessus la photographie. Photographie de Henry Balfour, 1922.

Avec l'aimable autorisation du Pitt Rivers Museum, University of Oxford 1998.547.1.
La création d'un nouveau cadre, dessiné par-dessus le mauvais cadrage d'origine, révèle les tensions inhérentes à ce que Deborah Poole a appelé «l'excès de description » de la photographie (Poole 2005), Balfour s'efforçant de contrôler tout ce qui, aux marges de celle-ci, détourne l'attention du sujet principal. Sur ses dessins, Balfour choisit ce qu'il représente sans y ajouter aucune espèce d'information ou de contexte; à l'inverse, la photographie possède bien un sujet central mais elle s'avère «bruyante » visuellement, c'est-à-dire incapable, dans l'environnement social et culturel désordonné de la rencontre sur le terrain, d'exclure les renseignements superflus. La pratique de Balfour nous permet également de mieux évaluer la manière dont ce qui est dessiné à la main interagit en permanence avec ce qui est enregistré par l'appareil photographique. Plutôt que de considérer la photographie et le dessin comme des méthodes parallèles ou concurrentes, il montre comment ces deux techniques peuvent servir de points de départ pour enregistrer des détails et produire des images susceptibles d'être reprises ensuite par un autre médium, reproduites, exposées ou diffusées.

Cela est particulièrement bien illustré par l'exemple suivant (fig. 8): sur une photographie extrêmement ternie, Balfour a dessiné les contours d'une femme en train d'allumer un feu par friction devant sa hutte. II a très vraisemblablement recopié l'élément dessiné et sans doute placé cette nouvelle image dans une vitrine du musée, à côté d'un archet servant à faire du feu. Sur l'image photographique imparfaite, Balfour a ajouté des contours tracés 
à la main, une pratique relativement commune à l'époque qui permettait d'utiliser des clichés de mauvaise qualité pris sur le terrain pour des expositions ou des publications. Mais Balfour fait plus qu'améliorer la qualité de la photographie: il crée par ces traits un objet intermédiaire, entre la saisie photographique directe sur le terrain et sa transformation en illustration ou image, destinée à être exposée publiquement pour contextualiser un objet du musée et montrer comment il est utilisé. On voit ici réactualisée une méthode de reproduction propre au $x \mathrm{XX}^{\mathrm{e}}$ siècle, celle des illustrations dont la légende spécifiait qu'elles étaient réalisées «à partir d'une photographie ", c'est-à-dire d'une image imparfaite passée au filtre du «jugement exercé» (Daston et Galison 2012).

\section{Kenya et Ouganda, 1928}

En juin 1928, Balfour se mit en route pour le Kenya puis l'Ouganda. Il avait déjà traversé ces pays à plusieurs reprises en se rendant en Afrique du Sud, mais ce fut sa première véritable expédition dans la région, à l'âge de soixante-cinq ans. Comme son voyage chez les Naga en 1922, celui-ci était motivé par un intérêt ethnographique de longue date, mais surtout par l'existence d'un réseau colonial qui lui permit d'organiser plus facilement ses différents trajets et activités de collecte. À la différence du journal qu'il tint chez les Naga, le carnet de 1928 contient des photographies et des dessins, probablement ajoutés à son retour en Angleterre. Balfour y colla par exemple une photographie de la maison d'Oscar Watkins, qui l'hébergea à Mombasa, ainsi que celle d'une crique voisine. Plus haut sur la page figure le dessin d'un corbeau à nuque blanche, qui, comme le note Balfour, prolifère dans la région. Sur une autre page, il a disposé la photographie de la Whispers Farm, propriété de la femme de Watkins où il passa une nuit: une petite pièce unique aux murs de briques et au toit de chaume. Au-dessus, on peut voir un petit dessin de la tête d'une femme africaine, que Balfour décrit dans son journal, à l'entrée du dimanche 22 juillet: «Fasciné par une infirmière indigène noire portant un uniforme d'infirmière + parée d'une double rangée bien dessinée de petites chéloïdes coniques sur le front. "Sur ces deux pages, comme ailleurs dans le carnet, la photographie et le dessin mêlent des registres différents, entre l'instantané de la vie coloniale et la description détaillée de l'observation ethnographique ou ornithologique. Balfour ne se sert pas du dessin pour saisir ou illustrer le contexte social et colonial de son voyage, la maison de ses hôtes par exemple. Cette fonction revient, comme c'était la convention alors, aux photographies instantanées, qui jouent le rôle de souvenirs. Balfour est un ethnographe qui utilise le dessin pour enregistrer les données de manière graphique, et non un dessinateur cherchant à rendre de manière artistique le monde qui l'entoure. Par conséquent, le choix de la méthode d'enregistrement des données est dicté par les possibilités descriptives de chaque médium en fonction de la situation. Cela est bien visible dans le cas des photographies montrant au loin des animaux et des oiseaux sauvages, collées à côté de dessins représentant deux espèces de gnous et une ombrette africaine. Balfour n'ayant pas réussi à s'approcher suffisamment pour faire des gros plans, les photographies servent seulement à authentifier les circonstances dans lesquelles il aperçut ces animaux et réalisa les dessins. Sur une page où il raconte sa rencontre avec des flamants roses sur le lac Nakuru, le 13 août, il écrit: «J'ai réussi à m'approcher des oiseaux + essayé 


\section{ci-contre}

fig. 9

Page 51 du carnet de terrain de Henry Balfou au Kenya et en Ouganda (1928). Pitt Rivers Museum Manuscript Collections, Balfour Papers, boîte 2 . Avec l'aimable autorisation du Pitt Rivers Museum, University of Oxford, Image ref: Balf.1928Keny \&Ugan.51-R. de les photographier", mais il ajoute aux photographies (sur lesquelles on distingue à peine les oiseaux) des dessins en couleurs et deux plumes collées sur la page.

Les interactions de Balfour avec les Africains furent ici, comme ailleurs au cours de ses voyages, facilitées par ses différents hôtes, membres de l'administration coloniale. Le 25 juillet, par exemple, il écrit:

Après le petit-déjeuner je me suis rendu dans le bureau de Deck + ai vu un nombre de Masai, que j'ai parfois pris en photo. Certains jouaient au mancala. J'ai pu voir de très bons types avec des traits très purs, d'autres présentaient un mélange avec les Kikuyu [...]. Un jeune Meru avait dans le lobe gauche un trou dans lequel il avait passé une boîte à cigarettes cylindrique en métal (pouvant contenir 50 cigarettes).

En face de cette description est présentée la photographie en question (fig. 9). Les hommes posent alignés, sans doute sur les ordres de Deck, l'hôte de Balfour ce matin-là, et de Hugo Lambert (un ancien étudiant de Balfour), I'hôte de l'après-midi, devant l'hôpital vétérinaire où l'ethnographe avait rencontré les deux groupes photographiés. En haut de la page, un dessin montre comment le jeune Meru porte la boîte à cigarettes. Plus tard, Balfour reproduisit ce croquis en grand format, qu'il ajouta aussi pendant un temps à l'entrée correspondante de son journal. Balfour réussit à obtenir l'objet de la part du jeune Meru - sans toutefois préciser comment. La boîte est aujourd'hui exposée au musée dans une vitrine consacrée au "recyclage" avec une autre, elle aussi obtenue par Balfour auprès d'un homme Nandi. Une autre copie du dessin est conservée à l'intérieur de la boîte. Si une grande partie des photographies de Balfour servent à contextualiser ses dessins d'observation, on trouve également une série très inhabituelle, prise en Ouganda, montrant les différentes étapes de fabrication d'une poterie. Même s'il était commun à l'époque de photographier les différentes étapes des rites ou les gestes techniques, Balfour s'intéressait avant tout à une comparaison interculturelle des formes finies plutôt qu'aux procédés de fabrication des objets eux-mêmes. Pour lui, la description graphique d'un procédé revêtait une importance capitale dans ce qu'elle montrait la manipulation de l'objet, et non l'inscription sociale ou culturelle de ce dernier dans une suite ou une chaîne de procédés de fabrication.

Le journal de Balfour en Afrique orientale met par ailleurs en lumière le lien décisif entre dessin et collecte d'objets. Sur une page du carnet (fig. 10) sont disposés plusieurs dessins: un Nandi avec des scarifications sur le visage, un autre homme portant un rouleau de papier à travers le lobe de l'oreille et le portrait de profil d'un «meurtrier Nandi» arborant un ornement à l'oreille. Les deux dessins en couleurs représentant des pendants d'oreille ont de toute évidence été ajoutés après avoir été collectés pour le musée. Par la suite, Balfour souhaita exposer ces pendants et les présenta dans une petite boîte vitrée accompagnée d'un dessin montrant la manière dont ils étaient portés. Le journal fournit ici des renseignements sur leur contexte d'acquisition et d'utilisation, tandis que le dessin sert de description précise permettant éventuellement de faire le lien entre tel ou tel objet et les informations collectées sur le terrain. Durant sa longue expérience de conservateur, Balfour travailla avec des objets qui n'étaient que rarement, voire jamais, accompagnés d'une description de terrain précisant le contexte de leur 


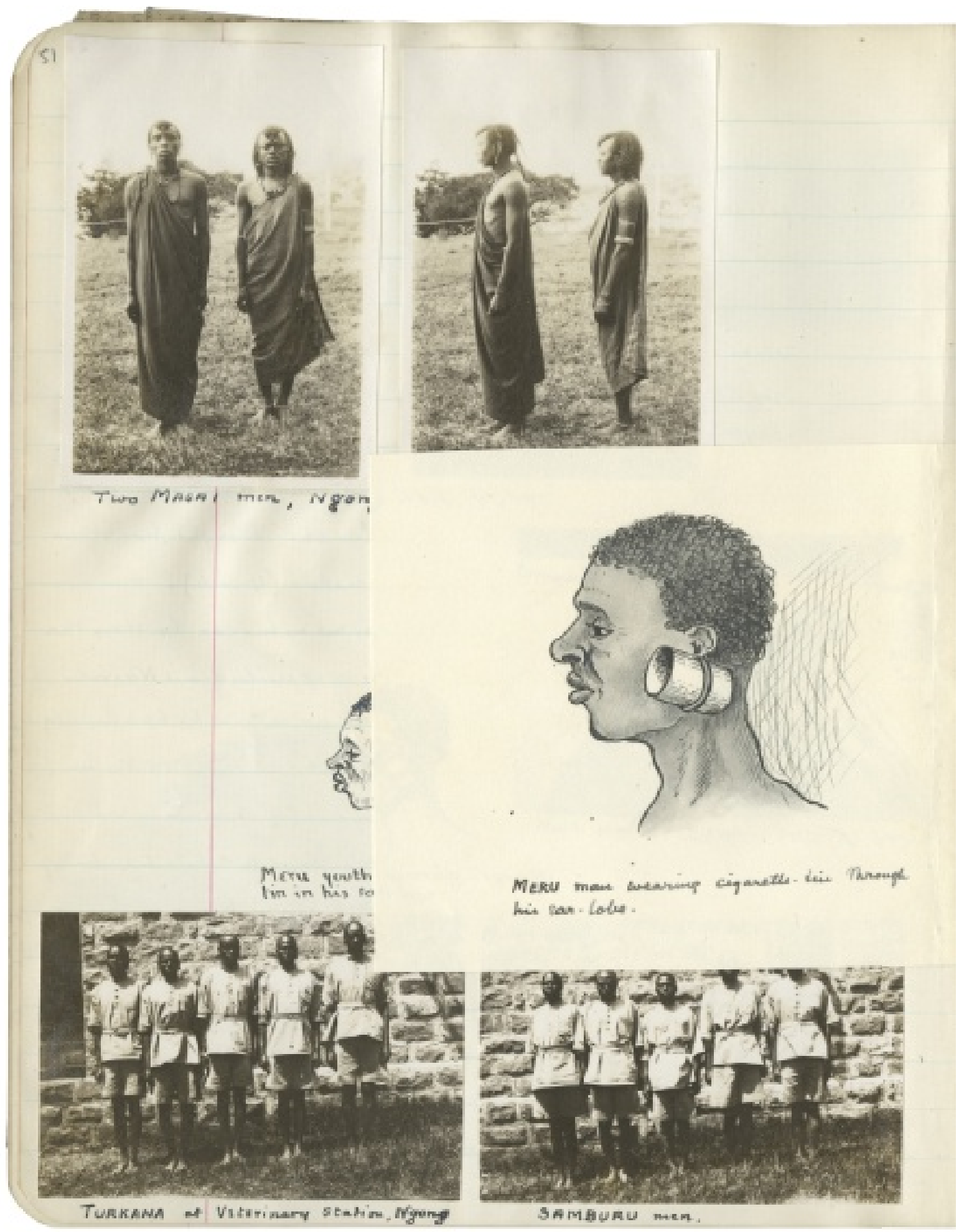




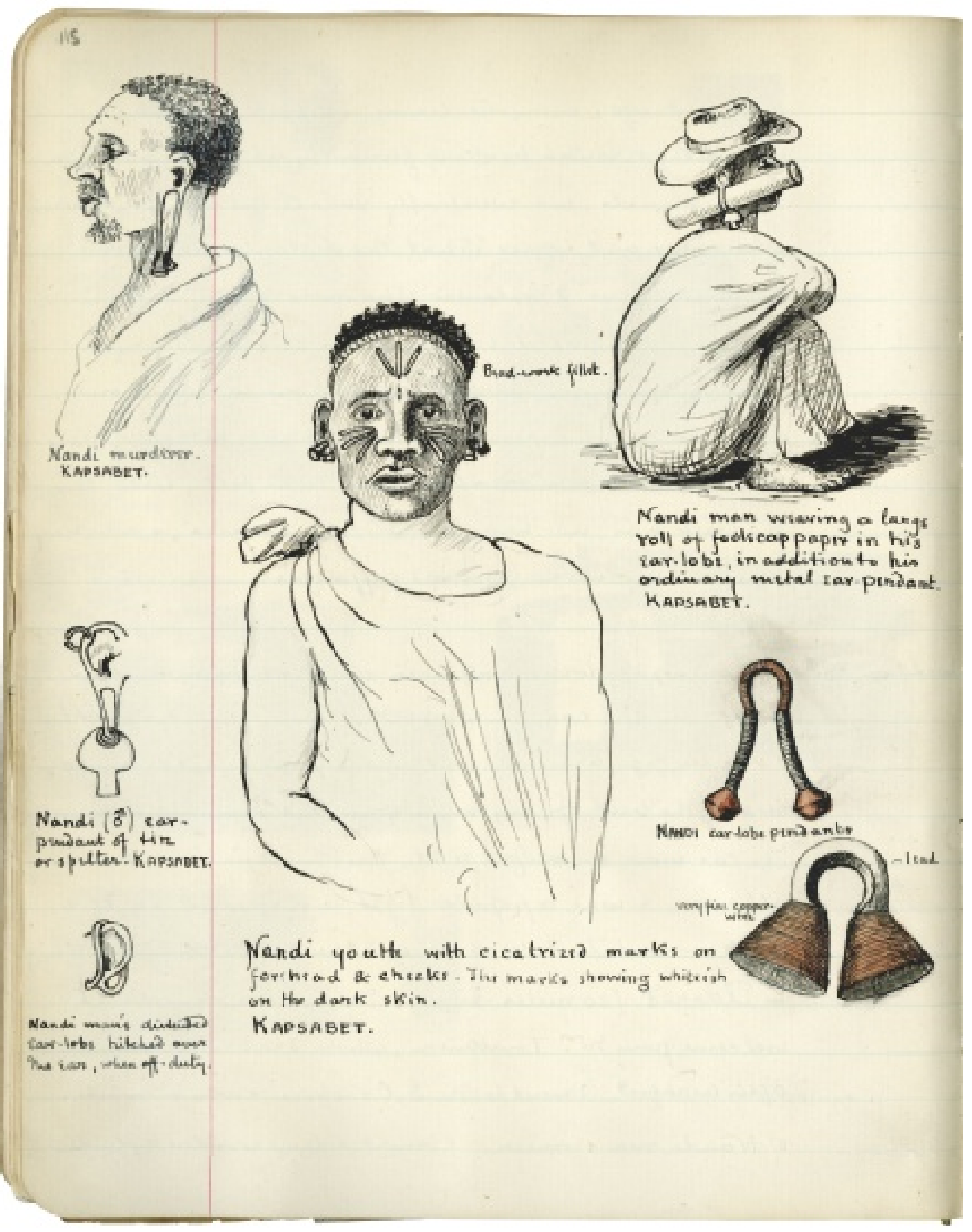


utilisation ou de leur collecte. Dès lors, sa manière de documenter très précisément les objets à partir d'observations de terrain servit de modèle méthodologique au sein du musée, au même titre que les renvois à ses carnets dans les registres, mentionnés plus haut.

\section{Anthropologie, dessin, exposition}

Un grand nombre de chercheurs se sont récemment penchés, d'un point de vue théorique, sur la pratique du dessin et de l'esquisse en anthropologie. Pour Tim Ingold, dessiner constitue le fondement d'une nouvelle «anthropologie graphique» mettant l'accent sur la phénoménologie, comme le montre l'ouvrage qu'il a dirigé, Redrawing Anthropology (Ingold 2011). À travers le dessin, l'observation est reconnectée à la participation, ainsi qu'à la description, puisque, dans le processus de connaissance, l'anthropologie est impliquée dans un acte de «fabrication" [making] et non pas d'imposition de structures analytiques sur le monde. De la même façon, Michael Taussig a cherché à relier la pratique du dessin à son intérêt de longue date pour la magie sympathique ${ }^{\mathbf{1 4}}$ : il établit ainsi un rapport entre le dessin en tant qu'acte de fabrication d'une image et son emprise sur celui qui dessine, comme sur celui qui regarde (Taussig 2011). À l'instar d'Ingold, Taussig veut réhabiliter le dessin comme manière anthropologique de se connecter au monde et de le connaître: c'est pour lui une façon d'entrer en contact avec des aspects inconnus et inexprimés, une «traduction inachevée» [incomplete translation]. Les deux anthropologues cités ici ont été influencés par les travaux de John Berger, qui a attiré l'attention sur les temporalités différentes du dessin et de la photographie. Si le dessin est un «faire», la photographie est, pour Berger, un «prendre", le premier relevant de la ligne, la seconde du cadre. Taussig reprend cette idée quand il suggère que le dessin contient une intimité, absente en photographie, un mouvement vers et une plongée dans le sujet. De la même façon, pour Ingold, le geste lié à l'appareil photographique est à l'exact opposé de ce qu'il propose: le dessin est pour lui affaire de nœuds, de maillage et d'assemblage, alors que la photographie impose un cadre extérieur. Berger invoque notamment le concept de trace pour décrire la relation entre la photographie et son référent. Ainsi, un dessin «contient le temps de sa fabrication", indépendant du temps de son sujet. À l'inverse, une photographie inscrit le temps de manière si rapide que «le seul temps que contient une photographie est l'instant isolé de ce qu'elle montre" (Berger et Mohr 2014: 93). Omar Nasim a lui aussi souligné la temporalité particulière de l'observateur qui se familiarise avec le sujet observé dans son étude sur les dessins d'observation dans l'histoire de l'astronomie (Nasim 2013). II remarque ainsi qu'«une familiarisation à ce point personnelle, viscérale et tactile permet de s'habituer (même avec un seul dessin) à ce qui est vu, à la manière de dessiner ce qui est vu et aux caractéristiques connues, inconnues et déroutantes d'un objet» (ibid: 16). Par ailleurs, Nasim insiste sur un autre aspect important du pouvoir des dessins d'observation: leur mutabilité en tant qu' "outils au service de l'exploration, du contrôle et de la perception", qui les distinguent des «mobiles immuables», par exemple les représentations photographiques (ibid: 10).

Tous ces cadres théoriques sont certes utiles pour comprendre l'usage que fait Balfour du dessin et de la photographie. Mais j'ai voulu montrer dans cet article que la fluidité à l'œuvre dans son travail entre l'acte de photographier et de dessiner, mais aussi entre les différents emplois de l'objet photographique
14. La magie sympathique fonctionne sur le principe de l'imitation et de la correspondance. Elle consiste à chercher à produire un effet à distance sur les choses par des phénomènes d'analogie. Un rituel peut ainsi procéder du même au même, du proche au proche, de la partie au tout: par exemple, les cheveux d'un individu valent pour l'individu tout entier. [NdT]

\author{
ci-contre \\ fig. 10 \\ Page 115 du carnet \\ de terrain de Henry Balfour \\ au Kenya et en Ouganda, \\ 1928. Pitt Rivers Museum \\ Manuscript Collections, \\ Balfour Papers, boîte 2. \\ Avec l'aimable autorisation \\ du Pitt Rivers Museum, \\ University of Oxford, \\ Image ref: Balf.1928Keny \\ \&Ugan.115-R.
}




\section{ci-contre}

fig: 11

Trois séries de notes de recherches prises par Henry Balfour sur la technique de la fabrication du feu. Pitt Rivers Museum Manuscript Collections, Balfour Papers, boîte 18, fichier 2. Avec l'aimable autorisation du Pitt Rivers Museum, University of Oxford. et du dessin, rend en réalité caduque toute distinction théorique. Comme l'ont justement souligné Anna Grimshaw et Amanda Ravetz (2015) à propos du cinéma, analyser séparément l'enregistrement effectué grâce à des appareils équipés de lentilles et le dessin, c'est ignorer la circulation historique entre ces pratiques en anthropologie. Pour Balfour, le dessin et la photographie partageaient des possibilités descriptives: la photographie pouvait par exemple servir à illustrer la manière de manipuler un artefact et être combinée avec des images de terrain, des dessins et des textes de sources diverses. C'est le cas notamment dans la recherche que mena Balfour sur les techniques de fabrication du feu: une page de carnet présente la photographie d'un bâton utilisé par les San (Bushmen), ici manié par un Européen, probablement dans une salle du musée, et, à côté, le dessin d'hommes San employant une technique semblable. Le dessin n'a pas été fait sur le vif ou à partir d'une photographie, mais «à partir d'une description ", ce qui souligne davantage encore, chez Balfour, les échanges permanents entre la preuve textuelle, dessinée et photographiée (fig: 11).

La notion de copie est, dans la pratique de Balfour, le lieu d'une grande tension. $\mathrm{Si}$, dans ses propres travaux sur l'évolution de l'art décoratif, il suggérait que la dégénérescence des formes naturelles en variantes stylisées pouvait s'expliquer par une série de copies imparfaites au fil du temps, il utilisait lui-même fréquemment le dessin pour copier des preuves visuelles à partir d'une variété de sources, dans le but de faire des comparaisons directes. Dans ses notes de recherche figure un cas particulièrement remarquable (fig. 12): Balfour recopia différents exemples des façons de percer le septum nasal, le premier à partir d'une gravure d'un livre de Friedrich Ratzel (en haut), le second s'inspirant d'une photographie dont la source n'a pas été identifiée (en bas à gauche) et le troisième reprenant une planche d'un article d'Alfred C. Haddon (en bas à droite). Ces différentes sources, placées ainsi sur le même plan, se retrouvent dotées d'une même légitimité, Balfour utilisant son «jugement exercé » pour identifier l'authenticité et la provenance de l'information. En les recopiant et en rassemblant ces trois illustrations sur une même page, Balfour exploite la capacité du dessin d'agir comme une technique de reproduction extrêmement labile dans le cadre scientifique du musée.

Le Pitt Rivers Museum explora en partie les possibilités de reproduction offertes par la photographie au début du xx $x^{e}$ siècle, en créant notamment des plaques de projection pour des séances éducatives ou en tirant des épreuves qui permettaient de partager certains objets du musée avec d'autres institutions et savants. De son côté, Balfour fut très actif dans la constitution et l'organisation d'archives photographiques à des fins de recherche (Morton 2012). Cependant, il préférait se fier à lui-même pour reproduire les preuves visuelles et illustrer l'utilisation des artefacts dans l'espace public du musée. Cela ne signifie pas qu'il ignorait les mises en garde scientifiques contre le biais artistique introduit dans les données anthropologiques. En effet, il veillait toujours à indiquer les sources de ses illustrations afin qu'elles puissent faire l'objet de vérifications éventuelles. Dans les cas où la source était une gravure ou un dessin fait sur le vif, il s'assurait du prestige scientifique de l'auteur. En 1931 et 1932, Balfour et son assistant entreprirent l'archivage systématique des photographies du musée classées par thème et par aire géographique (Morton 2012). En dépit des six cents épreuves et négatifs qui lui sont attribués, Balfour n'avait inclus dans ces archives spécifiques, distinctes de la collection 


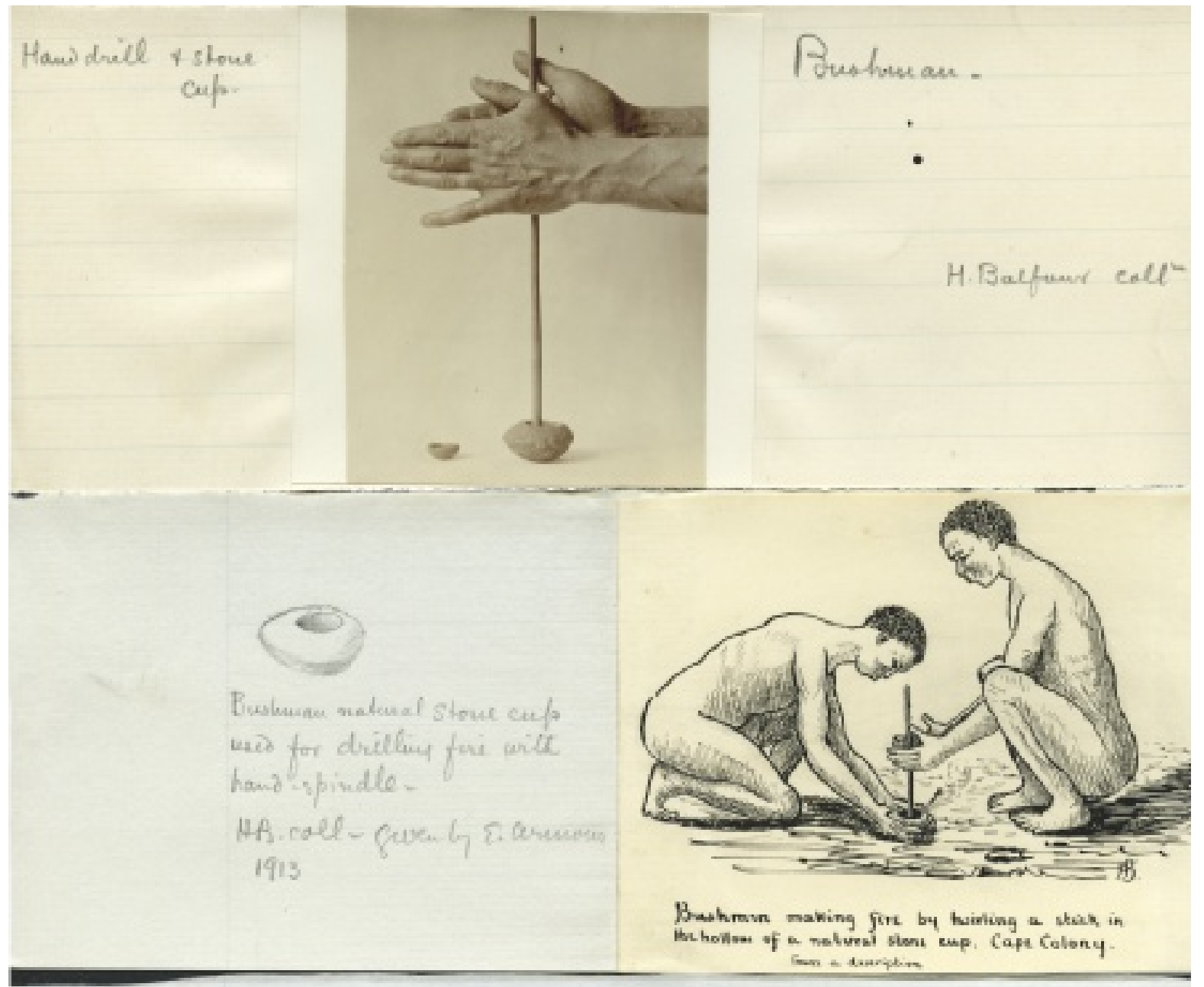

How driel

$$
\begin{aligned}
& \text { Yanādis, Madras } \\
& \text { E. Thurotou pust. }
\end{aligned}
$$

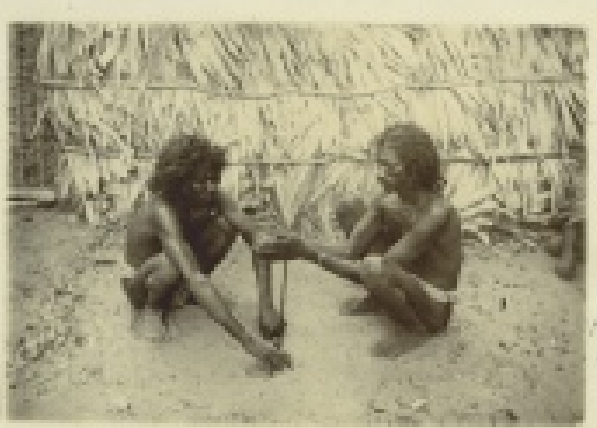




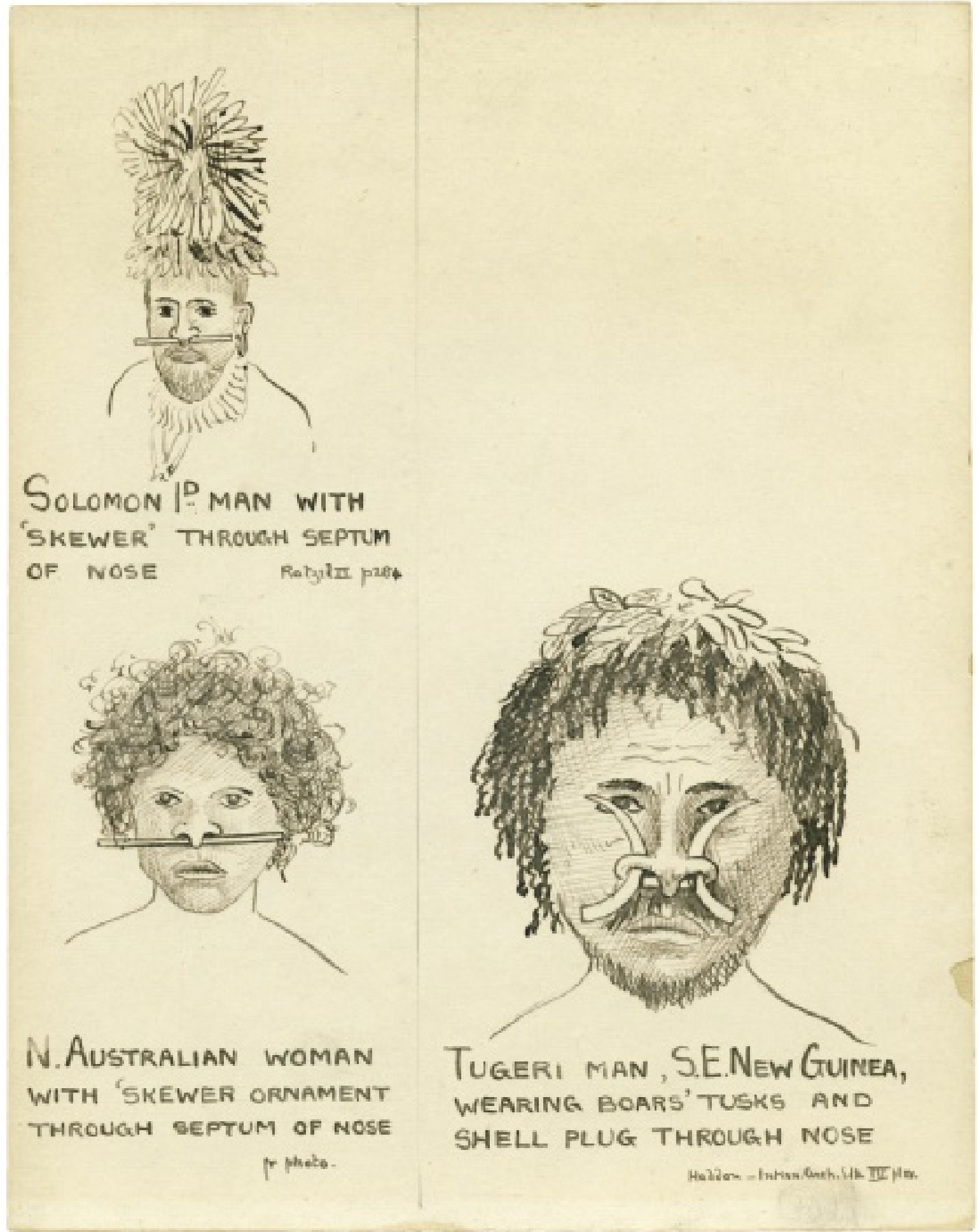


générale, que quarante épreuves, à titre d'échantillon, dont près de la moitié provenait de son voyage au Nigeria en 1930. II est difficile de savoir pourquoi il versa si peu de ses propres clichés aux archives photographiques du musée, mais cela correspond bien à son intérêt relativement limité pour ses photographies, par comparaison avec ses collections d'objets. Bien que certaines aient été collées dans ses journaux, beaucoup de négatifs et d'épreuves ne furent exhumés que plus tard, resurgissant parfois parmi les archives de collègues qui les avaient sans doute empruntés pour leur propre recherche ${ }^{15}$. D'autres fois, la renommée d'une revue suffisait à garantir le sérieux de ses sources, Balfour conservant très explicitement dans ses dessins de copie la nature photographique de l'original. Un exemple particulièrement parlant (fig. 13) est une composition (réalisée par l'assistant de Balfour au musée, Ernest $\mathrm{S}$. Thomas) de trois images issues de trois planches publiées par Gaston Muraz et Sophie Getzowa en 1923 dans la revue L'Anthropologie, auxquelles ont été ajoutées une échelle en pouces et une légende rédigée par Balfour. II est très vraisemblable que cette illustration ait été présentée dans une vitrine, à côté d'une paire de labrets de bois ${ }^{\mathbf{1 6}}$ en provenance du Tchad offerte au musée par l'administrateur français Jules Marcel de Coppet en 1929 (Muraz et Getzowa 1923). Ce choix montre que Balfour attribuait une valeur identique aux différents modes de représentation - la photographie, le dessin et l'objet matériel -, comme en témoigne toute sa pratique de conservateur.

\section{Conclusion}

En faisant des dessins à partir de photographies, Balfour libérait l'image de son existence temporelle et contextuelle spécifique, désormais capable de déployer ses possibilités illustratives dans l'espace du musée. Mais il lui arrivait aussi d'utiliser à l'occasion la reproductibilité de la technique photographique pour faire des copies de dessins, entrecroisant ainsi davantage les possibilités graphiques de ces deux médiums. Comme je l'ai montré dans le cas d'une photographie sur laquelle Balfour traça directement des contours, toute distinction trop rigide entre «faire» et «prendre», dessiner et photographier, n'aurait pas convenu à Balfour. Sa formation en sciences naturelles dans les années 1880 comprenait l'apprentissage du dessin d'observation en tant qu'instrument fondamental pour l'enregistrement précis de données. Comme l'écrivait l'auteur d'un compte rendu de l'ouvrage d'Edgar Crookshank, Photography of Bacteria [La Photographie de bactéries] en 1887, «le temps n'est pas encore venu de dire que [les photographies] ont supplanté des dessins précis et de bonne qualité » (cité in Tucker 2014: 394), un point de vue que Balfour aurait certainement partagé. Bien qu'il fût familier des débats sur la subjectivité et la précision de l'enregistrement non mécanique des données ethnographiques, il n'envisageait pas, étant donné sa formation et ses intérêts, d'obstacles théoriques entre l'observation et la saisie de ce qui est observé. En réalité, ces dernières n'étaient souvent qu'une seule et même chose, comme le montre le passage à la description graphique analysé dans cet article: l'information pouvait en effet circuler entre le texte, les dessins réalisés sur le vif (à partir de descriptions ou de photographies), les gravures (de dessins ou de photographies) publiées dans des livres, les photographies de terrain, les photographies de dessins ou de gravures, ou encore les plaques de projection faites à partir de dessins ou de
15. Les deux cents négatifs et épreuves issus de son voyage de 1922 au Nagaland, par exemple, ne réintégrèrent les collections du Pitt Rivers Museum qu'en 1997, lorsque le musée acquit une collection de documents d'archives appartenant à Heinrich Meinhard (1900-1975), un immigré allemand qui avait commencé à travailler au musée en 1938 et qui avait ensuite enseigné à l'université de Newcastle.

16. Numéro d'acquisition 1929.50.1. En 2017, il était encore possible de voir cette paire de labrets dans la galerie inférieure du musée consacrée aux arts du corps (vitrine 39A).

\section{ci-contre}

fig. 12

Notes de recherches de Henry Balfour sur les différents modes de piercing du septum nasal. Pitt Rivers Museum Manuscript Collections, Pitt Rivers Museum Papers, boîte 9, fichier 1 . Avec l'aimable autorisation du Pitt Rivers Museum, University of Oxford. 


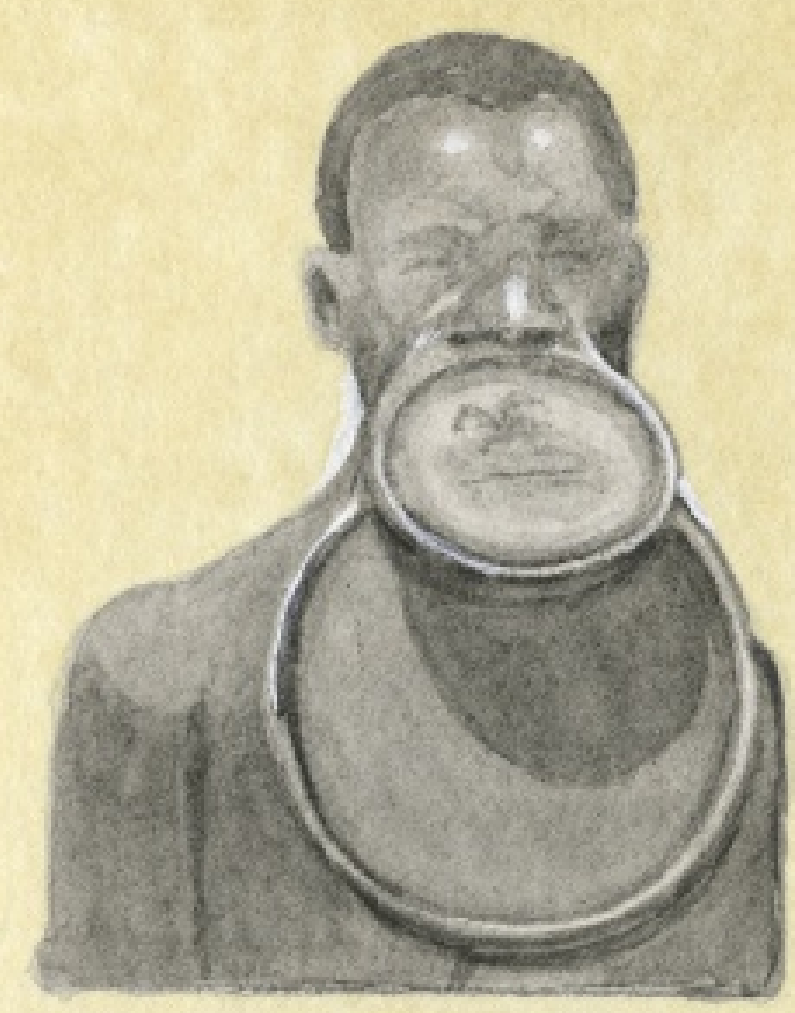

Old Sara woman with lipplate $24 \mathrm{~cm}$ ( $\left.9 \frac{1}{2} \mathrm{in}\right)$ in diameter P

The SARA JINGÉ, whose women wear these la the S.CHAD region. The French discourage [The plates are of a light wood.]
Sara women moveable by $t$ 


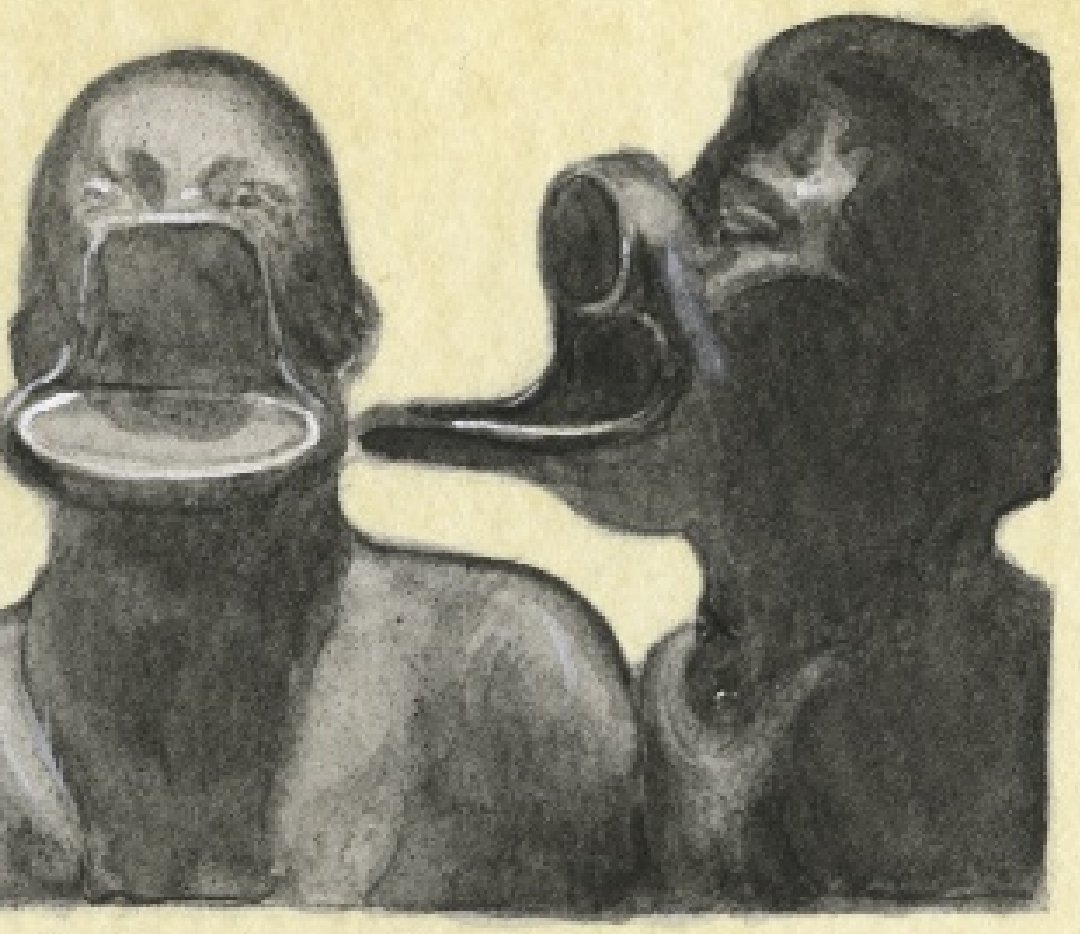

with lip plates still he lip muscles.

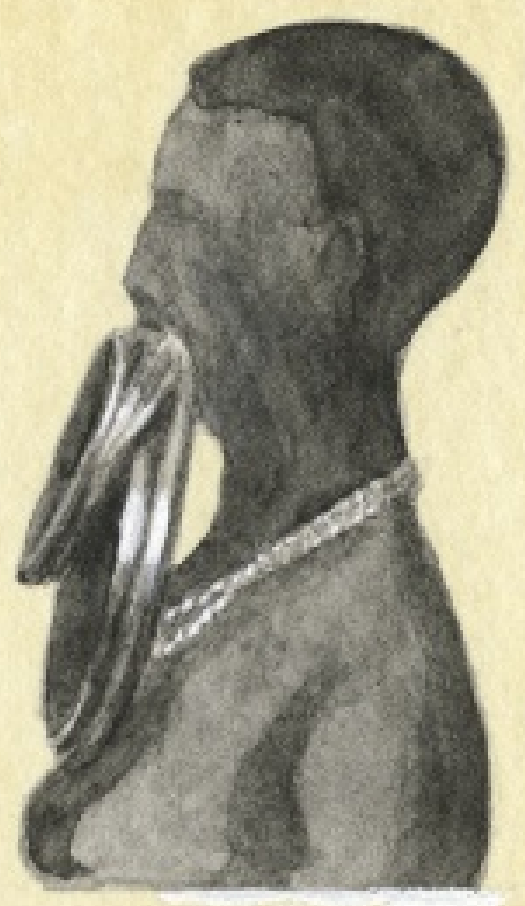

ES.T.

i

Side view of woman with very large plates.

ge lip plates ${ }^{*}$ are a branch of the SARA tribe, of the custom which is fast dying out.

[ZAnthropologie $x x \times 111,103$.] 


\section{double page précédente}

fig. 13

Dessin de Ernest $\mathrm{S}$

Thomas sur carton avec des notes de Henry Balfour sur les femmes Sara Kabba du Tchad portant des labrets, élaboré à partir de trois planches parues dans L'Anthropologie en 1923. Pitt Rivers Museum Manuscript Collections, Pitt Rivers Museum

Papers, boîte 9, fichier 1. Avec l'aimable autorisation du Pitt Rivers Museum,

University of Oxford. photographies. Les échanges entre tous ces différents médiums s'intensifièrent considérablement à partir des années 1880. L'approche comparative de Balfour pour l'étude de la culture matérielle accordait un rôle décisif à la copie et à la reproduction de données visuelles permettant de catégoriser, trier, archiver et formuler une argumentation. C'était avant tout le «jugement exercé» du spécialiste, sélectionnant des données faisant autorité dans des sources jugées fiables, qui déterminait la possibilité d'échanges fluides entre la photographie et le dessin sur le terrain, ou la réalisation de copies à partir de revues ou d'ouvrages pour étayer l'interprétation du musée. Balfour définissait ce travail de spécialiste dans l'ouvrage cité plus haut, Spinners and Weavers in Anthropological Research, où il convenait que:

Une grande responsabilité revient au «tisseur " de l'anthropologie, qui se doit d'être un artisan qualifié et ouvert d'esprit, ainsi qu'une personne capable de discernement, qui peut sélectionner et tester la résistance à la déformation et la solidité des fils que lui fournissent les «fileurs». (Balfour 1938: 8)

Comme je l'ai montré à propos des carnets de Balfour compilés au Nagaland et en Afrique orientale dans les années 1920, sa prédilection pour l'observation détaillée que requiert le dessin impliquait une temporalité spécifique, en accord avec sa formation et son approche. Berger souligne que le dessin contient sa propre temporalité pendant laquelle l'observateur refabrique l'objet de l'observation, tandis que la photographie incarne seulement l'acte de prendre l'image (Berger et Mohr 2014). Ceci explique évidemment pourquoi l'appareil photographique devint un outil si fréquemment utilisé dans le travail d'observation différé, c'est-à-dire postérieur à l'événement, quand les images prises sur le vif peuvent être examinées comme un carnet visuel bien après l'enquête de terrain. En ce sens, Balfour est typique des anthropologues de sa génération. Ainsi, on trouve dans le carnet de Haddon rapporté de sa mission sur l'île australienne de Mabuiag en 1888 une description qui rappelle à bien des égards la méthode de Balfour:

Ils sont très contents quand je leur montre des images de livres - ou mes carnets de dessins ou des épreuves grossières des photographies que j'ai prises d'eux... J'obtiens des descriptions de "costume" de danse, etc., en faisant des esquisses rapides d'un homme et en les remplissant petit à petit de détails, et ils sont absolument ravis quand ils ont la totalité devant les yeux. Parfois j'arrive à leur faire prendre un crayon pour qu'ils ajoutent eux-mêmes les détails. (Haddon 1888: 47-48)

L'usage combiné du dessin et de la photographie chez Haddon fait écho à l'approche de Balfour, la différence étant la profondeur des interactions avec les informateurs locaux sollicités. Comme le note Philp, les photographies de Haddon «participaient plutôt d'un travail en cours que d'une représentation finale » (Philp 2004: 95), un processus que j'ai décrit à propos de la circulation entre médiums dans le travail muséal de Balfour. Geismar remarque dans son analyse des dessins de Deacon au Vanuatu dans les années 1920 que "le dessin, au même titre que la photographie, peut se comprendre non seulement comme une manière de dépeindre des corps, mais aussi d'incarner un processus de connaissance qui contribue à donner à l'anthropologue 
un sens de l'échelle ou de sa position sur le terrain " (Geismar 2014: 105). Il est important en effet de se rappeler que l'acte de dessiner contient en lui-même des temporalités et des pratiques de terrain décisives: voir, observer, se positionner, participer et interroger le monde.

Formé aux sciences naturelles, Balfour transposa son engouement pour la morphologie animale à l'étude de l'«anatomie» de la culture matérielle. Pur produit intellectuel de l'université d'Oxford des années 1880, il est représentatif d'une démarche qui accordait un poids scientifique égal aux données textuelles, dessinées et photographiques et qui envisageait entre elles une grande fluidité. En ce sens, il est le reflet de son époque. Ainsi, les lettres rédigées par Franz Boas sur le terrain de la côte nord-ouest du Canada laissent parfois entrevoir ce lien entre le dessin et l'expérience de l'ethnographe: en novembre 1886, Boas écrit qu'il « [passa] la journée à dessiner, ce qui, étant donné le froid, n'est plus une occupation très agréable " (Rohner 1969: 59). Pour Balfour et ses contemporains, une photographie de terrain pouvait tout aussi bien servir de base à une illustration dessinée et bien nette, sans le bruit distrayant de l'époque historique et du lieu de création, la validité scientifique de l'image provenant du fait qu'elle avait été captée à un moment donné par l'appareil photographique. Dans le musée typologique, la question de l'histoire pouvait s'avérer problématique, puisque les objets étaient présentés dans une démarche comparative sans souci de temps ni d'espace, derrière une vitrine unique servant de cadre principalement anhistorique, que les objets fussent organisés selon un modèle évolutionniste de transformation ou d'hybridation des idées (c'était là une différence majeure entre Balfour et le schéma proposé par Pitt Rivers). En affranchissant le sujet central d'une photographie de sa dimension historique grâce au dessin, Balfour parvenait à réunir les objets matériels et les illustrations présentant leur contexte et leur usage au sein du schéma évolutionniste du musée, que les photographies tendaient précisément à déstabiliser en raison de leur inscription spécifique dans le temps et dans l'espace. 
Bibliographie

\section{Balfour, Henry}

1890 " On the Structure and Affinities of the Composite Bow", The Journal of the Anthropological Institute of Great Britain and Ireland 19: 220-250.

1893 The Evolution of Decorative Art: An Essay upon its Origin and Development as IIlustrated by the Art of Modern Races of Mankind. New York, Macmillan and Co.

1938 Spinners and Weavers in Anthropological Research. Oxford, Clarendon Press.

\section{Belknap, Geoffrey}

2016 From a Photograph: Authenticity, Science and the Periodical Press, 1870-1890. Londres, Bloomsbury.

\section{Berger, John et Mohr, Jean}

2014 Une autre façon de raconter. Paris, l'Écarquillé.

\section{Daston, Lorrain et Galison, Peter}

2012 Objectivité.

Paris, Les presses du réel.

\section{Douglas, Mary}

1995 "Acceptance", Science, Technology and Human Values 20 (2) : 262-265

\section{Geismar, Haidy}

2014 "Drawing it Out", Visual Anthropology Review 20 (2) : 97-113

\section{Gosden, Chris et Larson, Frances}

2007 Knowing Things: Exploring the Collections at the Pitt Rivers Museum, 1884-1945. Oxford, Oxford University Press

\section{Grimshaw, Anna et Ravetz, Amanda}

2015 «Drawing With a Camera? Ethnographic Film and Transformative Anthropology ", Journal of the Royal Anthropological Institute 21 (2): 255-275.

\section{Haddon, Alfred Cort}

1888 Torres Strait Fieldwork Journal (unpublished). Cambridge University Library, A. C. Haddon (HP) Papers

La description graphique. Dessins et photographies dans les carnets de terrain et le travail de conservateur de Henry Balfour. Par Christopher Morton

\section{Ingold, Tim (dir.)}

2011 Redrawing Anthropology:

Materials, Movements, Lines.

Farnham, Ashgate Publishing.

\section{Lang, Andrew}

1901 Myth, Ritual and Religion, t. II. Londres, Longmans, Green, and Co.

\section{Morton, Christopher}

$\mathbf{2 0 0 9}$ «Fieldwork and the Participant-Photographer: E. E. Evans-Pritchard and the Nuer Rite of Gorot ", Visual Anthropology 22 : 252-274.

2012 "Photography and the Comparative Method: The Construction of an Anthropological Archive ", Journal of the Royal Anthropological Institute 18 (2) : 369-396.

\section{Muraz, Gaston et Getzowa, Sophie}

1923 "Les lèvres des femmes Djingé, dites "Femmes-à-plateaux" ", L'Anthropologie 33: 103-125.

\section{Nasim, Omar}

2013 Observing by Hand: Sketching the Nebulae in the Nineteenth Century. Chicago, University of Chicago Press.

\section{Philp, Jude}

2004 " "Embryonic Science": The 1888 Torres Strait photographic Collection of A. C. Haddon", in Richard Davis (dir.), Woven Histories, Dancing Lives: Torres Strait Islander Identity, Culture and History. Canberra, Aboriginal Studies Press: 90-106.

\section{Poole, Deborah}

2005 "An Excess of Description: Ethnography, Race, and Visual Technologies ", Annual Review of Anthropology 34: 159-179.

\section{Rohner, Ronald}

1969 The Ethnography of Franz Boas: Letters and Diaries of Franz Boas Written on the Northwest Coast from 1886 to 1931. Chicago, University of Chicago Press.

\section{Taussig, Michael}

2011 / Swear / Saw This: Drawings in Fieldwork Notebooks, Namely My Own. Chicago, University of Chicago Press.

\section{Tucker, Jennifer}

2014 "Photography as Witness,

Detective, and Imposter: Visual

Representation in Victorian

Science ", in Bernard Lightman (dir.)

Victorian Science in Context.

Chicago, University of Chicago

Press: $378-408$.

\section{Tylor, Edward Burnett}

1871 Primitive Culture: Researches into the Development of Mythology, Philosophy, Religion, Language, Art and Custom, t. I.

Londres, John Murray.

\section{Wallis, Wilson Dallam}

1957 «Anthropology in England Early in the Present Century", American Anthropologist 59 (5) : 781-790. page 58 et ci-contre Trois séries de notes de recherches prises par Henry Balfour sur la technique de la fabrication du feu. Pitt Rivers Museum Manuscrip Collections, Balfour Papers, boîte 18, fichier 2. Avec l'aimable autorisation du Pitt Rivers Museum, University of Oxford. 
